\title{
AN EXTENSION THEOREM FOR CLOSING MAPS OF SHIFTS OF FINITE TYPE
}

\author{
JONATHAN ASHLEY
}

\begin{abstract}
If there exists some right-closing factor map $\pi: \Sigma_{A} \rightarrow \Sigma_{B}$ between aperiodic shifts of finite type, then any right-closing map $\varphi: X \rightarrow \Sigma_{B}$ from any shift of finite type $X$ contained in $\Sigma_{A}$ can be extended to a right-closing factor map from all of $\Sigma_{A}$ onto $\Sigma_{B}$. We prove this and give some consequences.
\end{abstract}

\section{INTRODUCTION}

We prove the following extension theorem for closing maps between shifts of finite type. In its statement, $\Sigma_{A}$ is the shift of finite type defined by the 0-1 matrix $A$, and $\left(G_{A}, \widehat{A}\right)$ is the dimension group pair associated to $\Sigma_{A}$ by Krieger [Kr1].

(1.1) Theorem. Let $A$ and $B$ be aperiodic 0-1 matrices satisfying.

(i) $A$ and $B$ have the same spectral radius.

(ii) The dimension group pair $\left(G_{B}, \widehat{B}\right)$ is a quotient of the dimension group pair $\left(G_{A}, \widehat{A}\right)$.

(iii) For each periodic point $p$ of $\Sigma_{A}$ there is a periodic point of $\Sigma_{B}$ whose period divides the period of $p$. (We denote this by $\Sigma_{A} \stackrel{\text { per }}{\rightarrow} \Sigma_{B}$. )

If $X \subset \Sigma_{A}$ is a subshift of finite type and $\tilde{\varphi}: X \rightarrow \Sigma_{B}$ is a right-closing map, then $\widetilde{\varphi}$ can be extended to a 1-to-1 almost everywhere right-closing map $\varphi: \Sigma_{A} \rightarrow \Sigma_{B}$.

Theorem (1.1) generalizes Theorem (3.15) of [A2] which is the special case where $X \subseteq \Sigma_{A}$ is a finite subshift. Our proof of Theorem (1.1) is a more intricate reworking of the proof of the case where $X \subseteq \Sigma_{A}$ is finite.

Theorem (1.1) also generalizes Theorem 6 of [KM] which is the special case where $\Sigma_{B}$ is the full $n$-shift. Following the proof of the main result of [KM], we prove the following corollary of Theorem (1.1).

(1.2) Theorem. Let $S$ be an aperiodic almost finite type sofic system, and let $\pi_{R}: \Sigma_{R} \rightarrow S$ be the canonical right-resolving cover of $S$ by a shift of finite type. Let $\Sigma_{B}$ be an aperiodic shift of finite type with the same entropy as $S$. Then the following two conditions are equivalent.

(i) The shift $S$ factors onto the shift $\Sigma_{B}$ by a 1-to-1 almost everywhere, rightclosing almost everywhere map.

Received by the editors December 18, 1990.

1980 Mathematics Subject Classification (1985 Revision). Primary 54H20, 58F11.

Supported by an NSF Postdoctoral Fellowship in the Mathematical Sciences. 
(ii) The dimension group pair $\left(G_{B}, \widehat{B}\right)$ is a quotient of the dimension group pair $\left(G_{R}, \widehat{R}\right)$; and $S \stackrel{\text { per }}{\rightarrow} \Sigma_{B}$.

Setting $\Sigma_{B}=\Sigma_{R}$ we get the following immediate corollary.

(1.3) Corollary. Let $S$ be an aperiodic almost finite type sofic system. Then the following are equivalent.

(i) $S$ is weakly isomorphic to its canonical right-resolving covering shift of finite type $\Sigma_{R}$.

(ii) $S \stackrel{\text { per }}{\rightarrow} \Sigma_{R}$.

In $\S 2$ we give some background; we prove Theorem (1.1) in $\S 3$, and prove Theorem (1.2) in $\S 4$. In $\S 5$ we give an example.

\section{BACKGROUND}

We assume some familiarity with shifts of finite type and their generalization, the sofic systems. Sections 2 and 3 of [BMT] are our main references for eventual factoring and dimension groups for shifts of finite type. Section 4 of [BMT] is our main reference for almost finite type sofic systems.

Given a 0-1 matrix $A$ indexed by a finite set $\mathscr{A}$, we define the shift of finite type $\Sigma_{A}$ by

$$
\Sigma_{A}=\left\{s \in \mathscr{A}^{\mathbb{Z}}: A_{s_{i} s_{i+1}}=1 \text { for } i \in \mathbb{Z}\right\}
$$

and topologize $\Sigma_{A}$ by the product of the discrete topologies on its coordinate spaces.

The 0-1 matrix $A$ also defines a directed graph $\mathscr{G}_{A}$ with states or symbols $\mathscr{A}$ and with a directed edge from state $s$ to state $t$ iff $A_{s t}=1$.

If $s_{1} s_{2} \cdots s_{k}$ is an allowable path of $k$ states in $\mathscr{G}_{A}$, we call $s_{1} s_{2} \cdots s_{k}$ a $k$-path of $\mathscr{G}_{A}$. Given a $k$-path $s_{1} s_{2} \cdots s_{k}$ of $\mathscr{G}_{A}$, we denote

$$
{ }_{n}\left[s_{1} s_{2} \cdots s_{k}\right]_{n+k-1}=\left\{x \in \Sigma_{A}: x_{n+i-1}=s_{i}, \quad 1 \leq i \leq k\right\} .
$$

This set is called a $k$-block of $\Sigma_{A}$. Given a set $\mathscr{S}$ of $k$-paths we denote

$$
{ }_{n}[\mathscr{S}]_{n+k-1}=\bigcup_{s \in \mathscr{S}}{ }_{n}[s]_{n+k-1} .
$$

Given $y \in \Sigma_{A}$ we denote the finite path $y_{i} y_{i+1} \cdots y_{j}$ in $\mathscr{G}_{A}$ by ${ }_{i}(y)_{j}$.

A $k$-block map $\pi: \Sigma_{A} \rightarrow \Sigma_{B}$ is a shift-commuting map such that there is some $l>0$ for which

$$
(\pi y)_{0}=\left(\pi y^{\prime}\right)_{0} \quad \text { if }{ }_{l-k+1}(y)_{l}=l-k+1\left(y^{\prime}\right)_{l} .
$$

The $k$-block map $\pi$ is a conjugacy if it is exactly 1-to-1. In this case $\pi^{-1}$ is an $m$-block map for some $m>0$.

In the 1-block case we require merely for notational convenience that $l=0$ in (2.1). In the 1-block case we have

$$
(\pi y)_{0}=\left(\pi y^{\prime}\right)_{0} \quad \text { if } y_{0}=y_{0}^{\prime} .
$$

Thus $\pi$ is defined by a map from single states of $\mathscr{G}_{A}$ to single states of $\mathscr{G}_{B}$ that we again call $\pi$.

A 1-block map $\pi: \Sigma_{A} \rightarrow \Sigma_{B}$ is right resolving if for every path $t_{1} t_{2}$ of length 2 in $\mathscr{G}_{B}$ and for every state $s_{1}$ of $\mathscr{G}_{A}$ with $\pi\left(s_{1}\right)=t_{1}$, there is at most one state $s_{2}$ such that $s_{1} s_{2}$ is an edge of $\mathscr{G}_{A}$ and $\pi\left(s_{2}\right)=t_{2}$. 
A $k$-block map $\pi: \Sigma_{A} \rightarrow \Sigma_{B}$ is right closing if it never identifies two distinct left asymptotic points: if $s, s^{\prime} \in \Sigma_{A}$ have an $l_{0} \in \mathbb{Z}$ such that $s_{l}=s_{l}^{\prime}$ for all $l \leq l_{0}$ and $\pi(s)=\pi\left(s^{\prime}\right)$, then $s=s^{\prime}$. Notice that a right-resolving map is right closing, and that the composition of two right-closing maps is again right closing.

A factor map is an onto block map, and a factor of a shift space is its image under a factor map.

A sofic system $S$ is a shift space (closed shift invariant subset of $\mathscr{B}^{\mathbb{Z}}$ for some finite alphabet $\mathscr{B}$ ) that is a factor of a shift of finite type [We].

A point $x$ in a shift space is left transitive if $\left\{\sigma^{n} x: n \leq 0\right\}$ is a dense subset of the space. A factor map $\varphi: X \rightarrow Y$ is right closing almost everywhere if it never identifies two left-asymptotic, left-transitive points [BMT].

We define (following [BMT]) the dimension group of integral matrix $A$ and collect here its properties that we use in this paper. If $A$ is an $\alpha \times \alpha$ integral matrix, let $V_{A}$ be the eventual range of $A$ regarded as a map $A: \mathbb{Q}^{\alpha} \rightarrow \mathbb{Q}^{\alpha}$ acting on row vectors. Thus $V_{A}=A^{\alpha}\left(\mathbb{Q}^{\alpha}\right)$. Define the dimension group

$$
G_{A}=\left\{\vec{q} \in V_{A}: \vec{q} A^{k} \in \mathbb{Z}^{\alpha} \text { for sufficiently large } k \in \mathbb{N}\right\},
$$

and the automorphism $\widehat{A}$ of $G_{A}$ by $\widehat{A}=\left.A\right|_{G_{A}}$. The dimension group $\left(G_{A}, \widehat{A}\right)$ associated to the shift of finite type $\Sigma_{A}$ is a conjugacy invariant.

(2.1) Proposition [BMT, Proposition 2.12]. Let $A$ and $B$ be integral eventually positive matrices with the same spectral radius, $\lambda$. Then the following are equivalent.

(1) $\left(G_{B}, \widehat{B}\right)$ is a quotient of $\left(G_{A}, \widehat{A}\right)$.

(2) There exists $L \geq 0$ and nonnegative integral matrices $S, R$ such that $A S=S B$ and $R S=B^{L}$.

(3) There exists $L \geq 0$ and positive integral matrices $S, R$ such that $A S=$ $S B$ and $R S=B^{L}$.

Let $A$ be an $\alpha$-by- $\alpha$ 0-1 matrix and $B$ be a $\beta$-by- $\beta$ 0-1 matrix. A rightresolving 1-block factor map $\pi: \Sigma_{A} \rightarrow \Sigma_{B}$ defines an $\alpha$-by- $\beta$ 0-1 matrix $S$ as follows:

$$
S_{i j}= \begin{cases}1 & \text { if } \pi(i)=j, \\ 0 & \text { otherwise }\end{cases}
$$

Because $\pi$ is right resolving, $A S=S B$. Notice that each row of $S$ has a single 1. Since $\pi$ is surjective, at least one 1 appears in each column of $S$, so there is a $\beta$-by- $\alpha$ matrix $R$ such that $R S$ is the $\beta$-by- $\beta$ identity matrix. Thus by Proposition $1.2\left(G_{B}, \widehat{B}\right)$ is a quotient of $\left(G_{A}, \widehat{A}\right)$.

Conversely if we are given $R, S$ such that $A S=S B$ and $R S=B^{L}$ and each row of $S$ is an elementary vector (all 0 's except one 1), then we immediately get a right-resolving factor map $\pi: \Sigma_{A} \rightarrow \Sigma_{B}$ defined by $\pi(s)=t$ iff $S_{s t}=1$.

It is more difficult to construct a factor map $\varphi: \Sigma_{A} \rightarrow \Sigma_{B}$ when the rows of $S$ are not all elementary vectors. It is still more difficult to construct a map $\varphi$ that extends a given right-closing map $\tilde{\varphi}: X \rightarrow \Sigma_{B}$ whose domain is a given shift of finite type contained in $\Sigma_{A}$. The construction uses a state-splitting algorithm that at each stage $i$ preserves the relation

$$
A^{(i)} S^{(i)}=S^{(i)} B, \quad 0 \leq i \leq n,
$$


for a sequence of matrices with $\Sigma_{A^{(i)}} \cong \Sigma_{A}$, where finally, $S^{(n)}$ has elementary rows. Moreover the right-resolving 1-block map $\mu: \Sigma_{A^{(n)}} \rightarrow \Sigma_{B}$ defined by $S^{(n)}$, when composed with the conjugacy $\Sigma_{A} \cong \Sigma_{A^{(n)}}$, gives an extension of the given $\operatorname{map} \tilde{\varphi}: X \rightarrow \Sigma_{B}$.

State splitting. Here we describe the state-splitting construction that we will use to conjugate the shift $\Sigma_{A}$ to a new shift $\Sigma_{A^{\prime}}$ moving closer to our goal.

Given a $0-1 \alpha$-by- $\alpha$ matrix $A$ and its associated directed graph $\mathscr{G}_{A}$, for each state $s$ in $\mathscr{G}_{A}$ denote by $\mathscr{F}(s)$ the set of states $t$ such that $s t$ is an edge of $\mathscr{G}_{A}$.

For each state $s_{i}, 1 \leq i \leq \alpha$, of $\mathscr{G}_{A}$ fix a partition $\mathscr{S}_{i}^{(1)}, \ldots, \mathscr{S}_{i}^{\left(r_{i}\right)}$ of the followers $\mathscr{F}\left(s_{i}\right)$ of $s_{i}$. We define a new graph $\mathscr{G}_{A^{\prime}}$ with states

$$
{ }_{0}\left[s_{i}\right]_{0} \cap{ }_{1}\left[\mathscr{S}_{i}^{(l)}\right]_{1}={ }_{0}\left[s_{i} \mathscr{S}_{i}^{(l)}\right]_{1}, \quad 1 \leq i \leq \alpha, \quad 1 \leq l \leq r_{i},
$$

and transitions (edges)

$$
{ }_{0}\left[s_{i} \mathscr{S}_{i}^{(l)}\right]_{1} \rightarrow{ }_{0}\left[s_{j} \mathscr{S}_{j}^{(m)}\right]_{l}
$$

if and only if $s_{j} \in \mathscr{S}_{i}^{(l)}$. We say that $\mathscr{G}_{A^{\prime}}$ is obtained from $\mathscr{G}_{A}$ by 1 round of state splitting.

The 1-block map $\alpha: \Sigma_{A^{\prime}} \rightarrow \Sigma_{A}$ given by $\alpha\left({ }_{0}\left[s_{i} \mathscr{S}_{i}^{(l)}\right]_{1}\right)=s_{i}$ is a conjugacy (an elementary conjugacy in the sense of [W]). In fact, the image under $\alpha$ of the 1 block ${ }_{0}\left[0\left[s_{i} \mathscr{S}_{i}^{(l)}\right]_{1}\right]_{0}$ in $\Sigma_{A^{\prime}}$ is the union of 2 blocks ${ }_{0}\left[s_{i} \mathscr{S}_{i}^{(l)}\right]_{1}$ in $\Sigma_{A}$.

One can prove by induction that if $\mathscr{G}_{A^{\prime}}$ is obtained from $\mathscr{G}_{A}$ by $m$ rounds of state splitting then the states of $\mathscr{G}_{A^{\prime}}$ can be identified with elements of a partition $\mathscr{P}_{1}, \mathscr{P}_{2}, \ldots, \mathscr{P}_{l}$ of the $(m+1)$-paths of $\mathscr{G}_{A}$ such that

(1) the states of $\mathscr{G}_{A^{\prime}}$ are ${ }_{0}\left[\mathscr{P}_{i}\right]_{m}, 1 \leq i \leq l$,

(2) for each $1 \leq i \leq l$, there is a state $s(i)$ of $\mathscr{G}_{A}$ such that ${ }_{0}\left[\mathscr{P}_{i}\right]_{m} \subseteq{ }_{0}[s(i)]_{0}$,

(3) there is a transition ${ }_{0}\left[\mathscr{P}_{i}\right]_{m} \rightarrow{ }_{0}\left[\mathscr{P}_{j}\right]_{m}$ iff ${ }_{0}\left[\mathscr{P}_{i}\right]_{m} \cap_{1}\left[\mathscr{P}_{j}\right]_{m+1} \neq \varnothing$,

(4) if ${ }_{0}\left[\mathscr{P}_{i}\right]_{m} \cap_{1}\left[\mathscr{P}_{j}\right]_{m+1} \neq \varnothing$ then ${ }_{1}\left[\mathscr{P}_{j}\right]_{m+1} \subseteq{ }_{1}\left[s(i)^{-1} \mathscr{P}_{i}\right]_{m}$, where $s(i)^{-1} \mathscr{P}_{i}$ is the set of $m$-paths $\left\{s_{j_{2}} s_{j_{3}} \cdots s_{j_{m+1}}: s(i) s_{j_{2}} s_{j_{3}} \cdots s_{j_{m+1}} \in \mathscr{P}_{i}\right\}$.

The 1-block map $\alpha: \Sigma_{A^{\prime}} \rightarrow \Sigma_{A}$ defined by $\alpha\left(0\left[\mathscr{P}_{i}\right]_{m}\right)=s(i)$ is a conjugacy we call the ancestor map after $[\mathrm{KM}]$. The state ${ }_{0}\left[\mathscr{P}_{i}\right]_{m}$ is a descendant of the state $s(i)$.

Legal splitting. Our starting point in constructing $\pi: \Sigma_{A} \rightarrow \Sigma_{B}$ is the set of equations $A S=S B$ and $R S=B^{L}$. In the sequel we denote the $i$ th row of any matrix $M$ by $M_{i, *}$ where we regard $M_{i, *}$ as a row vector. We think of the $\alpha$-by- $\beta$ matrix $S$ as assigning mass vectors $w(s)$ to the states $s$ of $\mathscr{G}_{A}$ by $w(s)=S_{s, *}$. Restating the equation $A S=S B$ in terms of mass vectors, we have for each state $s$ of $\mathscr{G}_{A}$

$$
\sum_{t \in \mathscr{F}(s)} w(t)=w(s) B
$$

We define a legal round of state splitting (legal with respect to $S$ ) of the graph $\mathscr{G}_{A}$ to be a round of state splitting in which for each state $s_{i} \in \mathscr{G}_{A}$, the followers $\mathscr{F}\left(s_{i}\right)$ of $s_{i}$ are partitioned into subsets $\mathscr{S}_{i}^{(1)}, \mathscr{S}_{i}^{(2)}, \ldots, \mathscr{S}_{i}^{\left(r_{i}\right)}$ in such a way that there are mass vectors $\vec{w}_{i}^{(1)}, \vec{w}_{i}^{(2)}, \cdots, \vec{w}_{i}^{\left(r_{i}\right)}$ with $\sum_{j=1}^{r_{i}} \vec{w}_{i}^{(j)}=w\left(s_{i}\right)$ and $\sum_{t \in \mathscr{S}_{i}^{(j)}} w(t)=\vec{w}_{i}^{(j)} B$ 
If the states of the graph $\mathscr{G}_{A^{\prime}}\left(\mathscr{G}_{\mathscr{A}^{\prime}}\right.$ being the graph obtained from $\mathscr{G}_{A}$ by splitting according to these partitions) are assigned mass vectors by

$$
w\left({ }_{0}\left[s_{i} \mathscr{S}_{i}^{(j)}\right]_{1}\right)=\vec{w}_{i}^{(j)},
$$

then condition (2.2) will remain true in $\mathscr{G}_{A^{\prime}}$; moreover some of the entries of $S^{\prime}$ (whose rows are the mass vectors $\vec{w}_{i}^{(j)}$ ) will be reduced in size from those of $S$.

Finally, a little more terminology. Given a graph $\mathscr{G}_{A}$ with state masses given by $S$, we call a state $s$ of $\mathscr{G}_{A}$ fat if $w(s)$ is not an elementary vector, and lean if it is. A fat path is a path in $\mathscr{G}_{A}$ having only fat states along it, and a fat cycle is a cycle having only fat states along it. An isolated fat cycle is a fat cycle $s_{0} s_{1} \cdots s_{p-1}$ such that each state $s_{i}$ on the cycle has only one fat successor: state $s_{i+1}$.

\section{Proof of the extension theorem}

In an attempt to motivate the following thicket of technical lemmas, we first outline the proof of Theorem (1.1).

First we show in Lemma (3.1) that we can assume without loss of generality that $\mathscr{G}_{A}, \mathscr{G}_{B}, X \subseteq \Sigma_{A}$, and $\widetilde{\varphi}: X \rightarrow \Sigma_{B}$ satisfy some hypotheses convenient to the construction of $\varphi: \Sigma_{A} \rightarrow \Sigma_{B}$. These are

(i) $X \subseteq \Sigma_{A}$ is presented as a subgraph $\mathscr{G}_{\widetilde{A}} \subseteq \mathscr{G}_{A}$,

(ii) $\tilde{\varphi}: \Sigma_{\tilde{A}} \rightarrow \Sigma_{B}$ is a right-resolving 1-block map,

(iii) the symbols of the sofic system $\widetilde{\varphi}(X)$ comprise the states of a proper subgraph $\mathscr{G}_{\widetilde{B}} \subseteq \mathscr{G}_{B}$,

(iv) each state $s$ of $\mathscr{G}_{\widetilde{A}}$ is followed in $\mathscr{G}_{A}$ by a state $t$ not in $G_{\widetilde{A}}$; similarly each state $s$ of $\mathscr{G}_{\widetilde{B}}$ is followed in $\mathscr{G}_{\widetilde{B}}$ by a state $t$ not in $\mathscr{G}_{\widetilde{B}}$. We say that $\mathscr{G}_{\widetilde{A}} \subseteq \mathscr{G}_{A}$ and $\mathscr{G}_{\widetilde{B}} \subseteq \mathscr{G}_{B}$ satisfy the escape hypothesis.

The extension $\varphi: \Sigma_{A} \rightarrow \Sigma_{B}$ of $\tilde{\varphi}: \Sigma_{\widetilde{A}} \rightarrow \Sigma_{\widetilde{B}}$ will be constructed by applying rounds of legal splitting to the graph $\mathscr{G}_{A}$ with state masses initially given by $S B^{N}$ for some $N>0$, to produce a graph $\mathscr{G}_{A^{\prime}}$ with all lean states. The map $\varphi: \Sigma_{A} \rightarrow \Sigma_{B}$ will then be the composition of the inverse ancestor map $\alpha^{-1}: \Sigma_{A} \rightarrow \Sigma_{A^{\prime}}$ with the 1-block mass map $\mu: \Sigma_{A^{\prime}} \rightarrow \Sigma_{B}$ defined by $\mu(s)=t$ where $w(s)=e_{t}$.

In order to ensure that $\varphi$ is an extension of $\widetilde{\varphi}$, the splittings leading from $\mathscr{G}_{A}$ to $G_{A^{\prime}}$ are controlled in such a way that the graph $\mathscr{G}_{A^{\prime}}$ contains as a subgraph, a graph isomorphic to the $(L+1)$-block graph $\mathscr{G}_{\widetilde{A}}^{[L+1]}$, for some $L>0$. The state $s$ in $\mathscr{G}_{A^{\prime}}$ identified with state ${ }_{0}\left[s_{0} s_{1} \cdots s_{L}\right]_{L}$ of $\mathscr{E}_{\widetilde{A}}^{[L+1]}$ is such that

$$
s \cap \Sigma_{\widetilde{A}}={ }_{0}\left[s_{0} s_{1} \cdots s_{L}\right]_{L} \cap \Sigma_{\widetilde{A}} .
$$

Here, $s$ is regarded as a subset of $\Sigma_{A}$, and $s_{0} s_{1} \cdots s_{L}$ is an $(L+1)$ path in $\mathscr{G}_{\widetilde{A}}$ (and in $\mathscr{G}_{A}$ ). The legal splittings leading to $\mathscr{G}_{A^{\prime}}$ are arranged so that $w(s)=e_{\tilde{\varphi}\left(s_{L}\right)}$ in $\mathscr{G}_{A^{\prime}}$. Now $s$ has ancestor $s_{0}$ in $\mathscr{G}_{A}$, so $\left.\left(\mu \circ \alpha^{-1}\right)\right|_{\tilde{A}}=\tilde{\varphi} \circ \sigma^{L}$. Thus, strictly speaking, it is $\varphi \circ \sigma^{-L}$ rather than $\varphi$ that is the extension of $\tilde{\varphi}$.

We divide the rounds of splitting leading from $\mathscr{G}_{A}$ to $\mathscr{G}_{\mathscr{A}}$, into two phases. The first phase, described in the proof of the Marker Splitting Lemma (3.4), eliminates all but a finite number of fat cycles. The second phase, described in the Isolated Cycle Lemma (3.6), eliminates the remaining fat cycles. 
(3.1) Lemma. By replacing $\Sigma_{A}$ and $\Sigma_{B}$ by conjugate shifts of finite type, we can assume without loss of generality that

(i) the shift of finite type $X \subseteq \Sigma_{A}$ is presented by a subgraph $\mathscr{G}_{\widetilde{A}}$ of the graph $\mathscr{G}_{A}$ with the property that an edge st of $\mathscr{G}_{A}$ is an edge of $\mathscr{G}_{\widetilde{A}}$ whenever $s$ and $t$ are both states of $\mathscr{G}_{\tilde{A}}$;

(ii) the map $\widetilde{\varphi}: \Sigma_{\widetilde{A}} \rightarrow \Sigma_{B}$ is a right-resolving 1-block map;

(iii) $\widetilde{\varphi}\left(\Sigma_{\widetilde{A}}\right) \subseteq \Sigma_{\widetilde{B}}$ where $\mathscr{G}_{\widetilde{B}}$ is a proper subgraph of $\mathscr{G}_{B}$;

(iv) $\mathscr{G}_{\widetilde{A}} \subseteq \mathscr{G}_{A}$ and $\mathscr{G}_{\widetilde{B}} \subseteq \mathscr{G}_{B}$ satisfy the escape hypothesis.

Proof. By the proof of Theorem 5 of [KM], we can assume (i) and (ii) without loss of generality. By replacing $\mathscr{G}_{A}$ with a higher order edge graph, we can assume that there is a cycle in $\mathscr{G}_{A}$ no state of which is in $\mathscr{G}_{\widetilde{A}}$.

We define a shift of finite type $\Gamma$ with $\Sigma_{\widetilde{A}} \subseteq \Gamma \subseteq \Sigma_{A}{ }^{A}$ and $\Gamma \neq \Sigma_{A}$ as follows. For each pair of states $(s, t)$ in $\mathscr{G}_{\tilde{A}}$, choose a path $u(s, t)$ in $\mathscr{G}_{A}$ such that $s u(s, t) t$ is a path in $\mathscr{G}_{A}$. For one pair of states $s, t$ in $\mathscr{G}_{\widetilde{A}}$ choose paths $u(s, t)$ and $v$ so that $s u(s, t) t$ and $s v t$ are both paths in $\mathscr{G}_{A}$ and $|v|=1+|u(s, t)|$. Let $\Gamma$ be the subshift of $\Sigma_{A}$ where $\Sigma_{\tilde{A}} \subseteq \Gamma$ and where the only allowed excursions possibly outside of $\mathscr{G}_{\widetilde{A}}$ are those along paths of the form $s u(s, t) t$ or $s v t$.

Now $\Gamma$ is a shift of finite type since one can determine whether or not $x$ is in $\Gamma$ by examining each $n$-word of $x$, where $n$ is the maximum length of the paths $s u(s, t) t$. We have $\Gamma \neq \Sigma_{A}$ since $\mathscr{G}_{A}$ has a cycle no state of which is in $\mathscr{G}_{\tilde{A}}$. Clearly $\Gamma$ is irreducible. We threw in the path svt to ensure that $\Gamma$ has positive entropy and is aperiodic.

By replacing $\mathscr{G}_{A}$ by a still higher order edge graph, we can assume that $\Gamma$ is presented by a subgraph $\mathscr{G}_{C}$ with $\mathscr{G}_{\tilde{A}} \subseteq \mathscr{G}_{C} \subseteq \mathscr{G}_{A}$. We can also assume that if $s, t$ are both states of $\mathscr{G}_{C}$ and $s t$ is an edge of $\mathscr{G}_{A}$, then st is an edge of $\mathscr{G}_{C}$. Let $\gamma$ be the number of states in $\mathscr{G}_{C}$, (so that $C$ is a $\gamma$-by- $\gamma$ matrix). Let $\vec{d}$ by the column vector with

$$
\begin{aligned}
d_{s}=\# \text { of followers of state } s \text { of } \mathscr{G}_{C} \text { that are not themselves } \\
\\
\text { in } \mathscr{G}_{C} .
\end{aligned}
$$

We define a splitting of $\mathscr{G}_{A}$ as follows:

(i) If state $s$ is not in $\mathscr{G}_{C}$, do not split $s$ at all;

(ii) If state $s$ is in $\mathscr{G}_{C}$, split $s$ according to the partition $\mathscr{P}_{0}, \mathscr{P}_{1}, \ldots, \mathscr{P}_{d_{s}}$ where $\mathscr{P}_{0}$ is the set of all followers of $s$ that are in $\mathscr{G}_{C}$, and each of $\mathscr{P}_{1}, \ldots, \mathscr{P}_{d_{s}}$ is a singleton set containing a distinct follower of $s$ not in $\mathscr{G}_{C}$.

If $\mathscr{G}_{A}$ is split according to this partition, then the resulting graph $\mathscr{G}^{(1)}$ will contain a subgraph isomorphic in the natural way to $\mathscr{G}_{C}$ (that we identify with $\mathscr{G}_{C}$ ). If we define the column vector $\vec{d}^{(1)}$ for $\mathscr{G}_{C} \subseteq \mathscr{G}^{(1)}$ as $\vec{d}$ was for $\mathscr{G}_{C} \subseteq \mathscr{S}_{A}$, then

$$
\vec{d}^{(1)}=C \vec{d} .
$$

If we repeatedly split $\mathscr{G}_{A}$ in this way, obtaining successive graphs $\mathscr{G}^{(1)}, \mathscr{G}^{(2)}$, 
$\ldots$, we obtain successive vectors $\vec{d}^{(i)}$

$$
\begin{aligned}
\vec{d}_{s}^{(i)}= & \# \text { of followers of state } s \text { in } \mathscr{G}_{C} \text { that are in } \mathscr{G}^{(i)} \text { but } \\
& \text { not in } \mathscr{G}_{C},
\end{aligned}
$$

and $\vec{d}^{(i)}=C^{i} \vec{d}$. Since $\Gamma$ is aperiodic, $C^{i}>0$ for large $i$. Thus $\mathscr{G}_{C}$, and hence $\mathscr{G}_{\widetilde{A}}$, satisfies the escape hypothesis in $\mathscr{G}^{(i)}$ for large $i$.

Similarly, by splitting $\mathscr{G}_{B}$ we can assume $\mathscr{G}_{\widetilde{B}} \subseteq \mathscr{G}_{B}$ satisfies the escape hypothesis.

Following [Kr2] denote by $\mathscr{N}[J, K], K \geq J$, the set of all $K$-paths $a_{1} a_{2} \ldots$ $a_{k}$ of $\mathscr{G}_{A}$ such that for every $j, 0<j<J$, there is at least one $k, 0<k \leq$ $K-j$, such that $a_{k} \neq a_{k+j}$.

(3.2) Lemma [Kr2, Lemma 2]. For all $J, K \in \mathbb{N}, K \geq J$, there is an $N \in \mathbb{N}$ and a finite union of $N$-blocks $F \subseteq \Sigma_{A}$ such that

$$
F \cap \sigma^{j} F=\varnothing, \quad 0<j<J,
$$

and

$$
\bigcup_{-J<j<J} \sigma^{j} F \supseteq \bigcup_{a \in \mathcal{N}[J, K]}{ }_{0}[a]_{k-1} .
$$

An easy corollary is

(3.3) Lemma. For all $J \in \mathbb{N}$, there is $N \in \mathbb{N}$ and a set of $N$-paths $\mathscr{B}$ such that

(1) No element of $\mathscr{B}$ nontrivially overlaps any other by more than $N-J$ states.

(2) The shift of finite type $\Sigma \subseteq \Sigma_{A}$ defined by forbidding occurences of $N$-paths in $\mathscr{B}$,

$$
\Sigma=\bigcap_{j=-\infty}^{\infty}\left(\Sigma_{A} \backslash j[\mathscr{B}]_{N+j-1}\right)
$$

consists of a finite number of periodic points, and these are exactly the periodic points of $\Sigma_{A}$ having period at most $J-1$.

Proof. Set $K=2 J-1$ and express the set $F$ of Lemma (3.2) as

$$
F=\bigcup_{i}\left[b^{(i)}\right]_{l+N-1}
$$

and let $\mathscr{B}$ be the set of $N$-paths $b^{(i)}$. Then

$$
\Sigma=\bigcap_{j=-\infty}^{\infty} \Sigma_{A} \backslash_{j}[\mathscr{B}]_{j+N-1} \subseteq \bigcap_{j=-\infty}^{\infty} \bigcap_{a \in \mathcal{N}[J, 2 J-1]} \Sigma_{A} \backslash_{j}[a]_{j+2 J-2} .
$$

Thus if $x \in \Sigma$, then for each $(2 J-1)$-path $w$ of $x$ there is a $j, 0<j<J$, such that $w$ is $j$-periodic. Now suppose $2 J-1 \leq L$ and that every $L$ path $w$ of $x$ is $j$-periodic for some $j<J, j$ perhaps depending on $w$. We show inductively that every $(L+1)$-path $v$ of $x$ is $j$-periodic for some $j<J, j$ perhaps depending on the $(L+1)$-path $v$. Let $s_{1} s_{2} \cdots s_{L+1}$ be an $(L+1)$ path from $x$. Now $s_{1} s_{2} \cdots s_{L}$ is $j$ periodic for some $j<J$ and $s_{2} s_{3} \cdots s_{L+1}$ is $k$ periodic for some $k<J$. Now $s_{L+1}=s_{L+1-k}$ because 
$s_{2} s_{3} \cdots s_{L+1}$ is $k$ periodic; $s_{L+1-k}=s_{L+1-k-j}$ because $s_{1} s_{2} \cdots s_{L}$ is $j$ periodic and $L+1-k-j \geq 2>1 ; s_{L+1-k-j}=s_{L+1-j}$ because $s_{2} s_{3} \cdots s_{L+1}$ is $k$ periodic and $L+1-k-j \geq 2$. Thus, $s_{L+1}=s_{L+1-j}$ so $s_{1} s_{2} \cdots s_{L+1}$ is $j$ periodic. It follows by induction that any finite path from $x$, and hence $x$ itself, is $j$ periodic for some $j<J$. Thus $\Sigma$ contains only periodic points of period less than $J$.

On the other hand, since $F \cap \sigma^{j} F=\varnothing, 0<j<J$, none of the $N$ paths in $\mathscr{B}$ is $j$ periodic for any $0<j<J$. Thus none of the periodic points of period less than $J$ in $\Sigma_{A}$ is removed by disallowing the $N$-paths in $\mathscr{B}$. Thus $\Sigma$ consists of exactly the periodic points of $\Sigma_{A}$ of period less than $J$.

(3.4) Marker Splitting Lemma. Let $A$ and $B$ be aperiodic 0-1 matrices and let $R, S$ be positive matrices and $l>0$ satisfy $A S=S B$ and $R S=B^{l}$. Let $\mathscr{G}_{\widetilde{A}}$ be a subgraph of $\mathscr{G}_{A}$ and let $\mathscr{G}_{\widetilde{B}}$ be a subgraph of $\mathscr{G}_{B}$, both subgraphs satisfying the escape hypothesis, and let $\widetilde{\varphi}: \Sigma_{\widetilde{A}} \rightarrow \Sigma_{\widetilde{B}}$ be any right-resolving 1-block map. Then there is $L>0$ such that the graph $\mathscr{G}_{A}$ with mass vectors given by $S B^{L}$ can be split by a finite number of rounds of legal splittings into a graph $\mathcal{G}^{\prime}$ having the following properties.

(1) If $s$ is a state of $\mathscr{G}^{\prime}$ and $s \cap \Sigma_{\widetilde{A}} \neq \varnothing$, then $s \cap \Sigma_{\widetilde{A}}$ is a union of $(L+1)$ blocks in $\Sigma_{\widetilde{A}}$ and

$$
w(s) \geq \Sigma\left[e_{\widetilde{\varphi}\left(s_{L}\right)}:{ }_{0}\left[s_{0} s_{1} \cdots s_{L}\right]_{L} \cap s \cap \Sigma_{\widetilde{A}} \neq \varnothing\right] .
$$

(2) $\mathscr{G}^{\prime}$ has only a finite number of fat cycles.

(3) The states along a fat cycle $\mathscr{C}$ in $\mathscr{G}^{\prime}$ are $(L+1)$-blocks ${ }_{0}\left[s_{0} s_{1} \cdots s_{L}\right]_{L} \subseteq$ $\Sigma_{A}$, where the path $s_{0} s_{1} \cdots s_{L}$ is $j$ periodic for some $j<J$.

(4) The mass of a state $s={ }_{0}\left[s_{0} s_{1} \cdots s_{L}\right]_{L}$ on a fat cycle $\mathscr{C}$ in $\mathscr{G}^{\prime}$ is $w(s)=$ $S_{s_{L}, *}$.

(5) Each fat cycle $\mathscr{C}$ in $\mathscr{G}^{\prime}$ is isolated.

Proof. In the proof of the Eventual Factors Theorem [BMT], it is shown that the entries of the matrix $R A^{n}$ grow exponentially in $n$ with growth rate $\lambda$, the spectral radius of $A$ (and $B$ ); while the magnitude of the entries of the matrix $A^{l+n}-S R A^{n}$ grow exponentially in $n$ with growth rate that is strictly less than $\lambda$. Thus there is $n_{0}>0$ such that for $n \geq n_{0}$,

$$
e_{1} R A^{n}+e_{S}\left(A^{l+n}-S R A^{n}\right)>0
$$

for each state $s$ in $\mathscr{G}_{A}$.

Still following [BMT], it follows from a matrix calculation that for any $n>$ $n_{0}$, the set of $J$ paths (where $J=l+n$ ) in $\mathscr{G}_{A}$ with initial state a follower of state $s$ can be partitioned into subsets $\mathscr{S}(s ; i, j), 1 \leq i \leq \beta, 1 \leq j \leq S_{s, i}$, such that

$$
\Sigma\left[e_{d_{J}}: d_{1} d_{2} \cdots d_{J} \in \mathscr{S}(s ; i, j)\right]=e_{i} R A^{n} \quad \text { if } i \neq 1 \text { or } j \neq 1
$$

and

$$
\Sigma\left[e_{d_{J}}: d_{1} d_{2} \ldots d_{J} \in \mathscr{S}(s ; 1,1)\right]=e_{1} R A^{n}+e_{s}\left(A^{J}-S R A^{n}\right) .
$$

It follows from $\left(A^{J}-S R A^{n}\right) S=0$ that

$$
\Sigma\left[S_{d_{J}, *}: d_{1} d_{2} \cdots d_{J} \in \mathscr{S}(s ; i, j)\right]=e_{i} B^{J} .
$$

Here, we further require that if $s$ is a state of $\mathscr{G}_{\tilde{A}}$, then the set of $J$ paths $\mathscr{S}(s ; \widetilde{\varphi}(s), 1)$ contains all of the $J$ paths in $\mathscr{G}_{\widetilde{A}}$ with initial state a follower of 
state $s$. We show that this is possible for sufficiently large $J$ as follows. The spectral radius of $\widetilde{A}$ is less than that of $A$, so for sufficiently large $n$

$$
e_{s} \widetilde{A}^{l+n} \leq \begin{cases}e_{\widetilde{\varphi}(s)} R A^{n} & \text { if } \tilde{\varphi}(s) \neq 1, \\ e_{1} R A^{n}+e_{s}\left(A^{l+n}-S R A^{n}\right) & \text { if } \tilde{\varphi}(s)=1,\end{cases}
$$

for each state $s$ of $\mathscr{G}_{\widetilde{A}}$. Now

$$
\Sigma\left[e_{d_{J}}: s d_{1} d_{2} \cdots d_{J} \text { is a path in } \mathscr{G}_{\widetilde{A}}\right]=e_{s} \widetilde{A}^{J}=e_{s} \widetilde{A}^{l+n},
$$

so for sufficiently large $J$, we can choose $\mathscr{S}(s ; \tilde{\varphi}(s), 1)$ as described.

Fix such a $J$ and such partitions. For this $J$, fix a set of $N$ paths $\mathscr{B}$ as in Lemma (3.3).

We will specify $N+2 J-2$ rounds of legal splitting applied to the graph $\mathscr{G}_{A}$ with initial mass vectors given by $S B^{N+J-2}$. But first we will present the graph $\mathscr{H}$ resulting from these splittings. The graph $\mathscr{H}$ satisfies properties (1)-(4), but not necessarily (5); some fat cycles of $\mathscr{H}$ might not be isolated. Later, we will further split $\mathscr{H}$ to isolate the fat cycles while preserving properties (1)-(4).

States of $\mathscr{H}$ :

$$
0[c]_{N+J-2}
$$

where $c$ is an $(N+J-1)$-path of $\mathscr{G}_{A}$ such that no $N$-path $b \in \mathscr{B}$ occurs in $c$.

$$
{ }_{0}\left[b \mathscr{S}\left(b_{N} ; i, j\right)\right]_{N+J-1}
$$

where $b \in \mathscr{B}$ and $1 \leq i \leq \beta, 1 \leq j \leq S_{b_{N}, i}$.

$$
{ }_{0}\left[d b \mathscr{S}\left(b_{N} ; i, j\right)\right]_{N+J-1+|d|},
$$

where $b \in \mathscr{B}$ and $d b$ is a $(|d|+N)$ path in $\mathscr{G}_{A}$ with $|d| \leq J-1$, and $i, j$ are as in (2).

Transitions of $\mathscr{H}$ :

$$
{ }_{0}[c]_{N+J-2} \rightarrow{ }_{0}[d]_{N+J-2},
$$

if $d_{1} d_{2} \cdots d_{N+J-2}=c_{2} c_{3} \cdots c_{N+J-1}$ and $d_{J} d_{J+1} \cdots d_{J+N-1} \notin \mathscr{B}$.

$$
{ }_{0}[c]_{N+J-2} \rightarrow{ }_{0}\left[d b \mathscr{S}\left(b_{N} ; i, j\right)\right]_{N+2 J-2},
$$

if $c_{2} c_{3} \cdots c_{N+J-1}=d b_{1} b_{2} \cdots b_{N-1}$ and $b=b_{1} b_{2} \cdots b_{N} \in \mathscr{B}$.

$$
{ }_{0}\left[b \mathscr{S}\left(b_{N} ; i, j\right)\right]_{N+J-1} \rightarrow{ }_{0}[c]_{N+J-2} \text {, }
$$

if $c$ is an $(N+J-1)$ path in $\mathscr{G}_{A}$ such that $b_{1} c \in b \mathscr{S}\left(b_{N} ; i, j\right)$ and no element of $\mathscr{B}$ occurs as a suffix of $c$ (which is the earliest possible such occurrence).

$$
{ }_{0}\left[b \mathscr{S}\left(b_{N} ; i, j\right)\right]_{N+J-1} \rightarrow{ }_{0}\left[d b^{\prime} \mathscr{S}\left(b_{N}^{\prime} ; i^{\prime}, j^{\prime}\right)\right]_{N+2 J-2},
$$

if $d b^{\prime}$ is an $(N+J-1)$ path such that $b_{1} d b^{\prime} \in b \mathscr{S}\left(b_{N} ; i, j\right)$ and $b^{\prime} \in \mathscr{B}$.

$$
{ }_{0}\left[d b \mathscr{S}\left(b_{N} ; i, j\right)\right]_{N+J-1+|d|} \rightarrow{ }_{0}\left[d^{\prime} b \mathscr{S}\left(b_{N} ; i, j\right)\right]_{N+J-2+|d|},
$$

where $d=d_{1} d^{\prime} \quad\left(d^{\prime}\right.$ may be the empty string).

Masses of states of $\mathscr{H}$ :

$$
\begin{gathered}
w\left({ }_{0}[c]_{N+J-2}\right)=S_{C_{N+J-1}, *}, \\
w\left({ }_{0}\left[b \mathscr{S}\left(b_{N} ; i, j\right)\right]_{N+J-1}\right)=e_{i} B^{J-1}, \\
\left.w{ }_{0}\left[d b \mathscr{S}\left(b_{N} ; i, j\right)\right]_{N+J-1+|d|}\right)=e_{i} B^{J-1-|d|} .
\end{gathered}
$$


Now we verify that $\mathscr{H}$ with its mass vectors can be obtained by legally splitting $\mathscr{G}_{A}$ with its mass vectors given by $B S^{N+J-2}$. Set $L=N+J-2$. The rounds of splitting are divided into four stages.

1. Perform $N-1$ rounds of complete splitting (at each round the set of followers of a state is partitioned into singleton sets), yielding the graph whose states are the $N$-blocks of states of $\mathscr{G}_{A}$. If after each round $j, 1 \leq j \leq N-1$, the state ${ }_{0}\left[s_{1} s_{2} \cdots s_{j+1}\right]_{j}$ is assigned mass vector $S_{s_{j+1}, *} B^{L-j}$ then each of these rounds is legal.

2. Perform $J-1$ rounds of splitting, completely splitting every state except the states ${ }_{0}[b]_{N-1}, b \in \mathscr{B}$, which are not split at all in any of the $J-1$ rounds. Call the resulting graph $\mathscr{G}_{1}$. After the $j$ th of these $J-1$ rounds the states of the graph are the blocks

$$
\begin{gathered}
{ }_{0}[b]_{N-1}, \quad b \in \mathscr{B} \\
{ }_{0}[c b]_{N-1+i},
\end{gathered}
$$

where $b \in \mathscr{B}, 0<i \leq j$, and $c$ is an $i$ path in $\mathscr{G}_{A}$ such that $c b$ is an $(N+i)$ path in $\mathscr{G}_{A}$,

$$
0[c]_{N-1+j}
$$

where $c$ is an $(N+j)$ path in $\mathscr{G}_{A}$ such that no path $b \in \mathscr{B}$ is a subpath of $c$. If after the $k$ th of these $J-1$ rounds the states are assigned masses by

$$
\begin{gathered}
w\left({ }_{0}[b]_{N-1}\right)=S_{b_{N}, *} B^{J-1}, \quad b \in \mathscr{B}, \\
w\left({ }_{0}[c b]_{N-1+i}\right)=S_{b_{N}, *} B^{J-1-i}, \quad 1 \leq i \leq k, \\
w\left({ }_{0}[c]_{N-1+k}\right)=S_{c_{N+k}, *} B^{L-(N-1+k)},
\end{gathered}
$$

then each of these rounds is legal.

3. Perform the following round of splitting. For $b \in \mathscr{B}$, split ${ }_{0}[b]_{N-1}$ into the states ${ }_{0}\left[b \mathscr{S}\left(b_{N} ; i, j\right)\right]_{N+J-1}$ for $1 \leq i \leq \beta$ and $1 \leq j \leq S_{b_{N}, i}$. Assign the displayed state the mass vector $e_{i} B^{J-1}$.

We show that this splitting is possible and legal. If $c=c_{1} c_{2} \cdots c_{N+J-1}$ is a path in $\mathscr{G}_{A}$ with $c_{1} c_{2} \cdots c_{N-1}=b_{2} b_{3} \cdots b_{N}$, then no $N$ path $b^{\prime} \in \mathscr{B}$ can begin in $c$ before position $J$, so ${ }_{0}[c]_{N+J-2}$ is a state of $\mathscr{G}_{1}$ following state ${ }_{0}[b]_{N-1}$. Hence we can partition the followers of ${ }_{0}[b]_{N-1}$ into the subsets

$$
\left\{0[c]_{N+J-2}: c_{N} c_{N+1} \cdots c_{N+J-1} \in \mathscr{S}\left(b_{N} ; i, j\right)\right\} .
$$

Now the mass of the state ${ }_{0}[c]_{N+J-2}$ in $\mathscr{G}_{1}$ is $S_{c_{N+J-1}, *}$, so the total mass of the elements of the above subset specified by $\mathscr{S}\left(b_{N} ; i, j\right)$ is $e_{i} B^{J}$ by the choice of the set of $J$ paths $\mathscr{S}\left(b_{N} ; i, j\right)$ given by the Eventual Factors Theorem. Thus state ${ }_{0}[b]_{N-1}$ can be legally split as described.

4. Perform the following $J-1$ rounds of splitting. At the $k$ th of these $J-1$ rounds, split the states ${ }_{0}[d b]_{N-1+k}$, where $b \in \mathscr{B}$ and $|d|=k$ into the states ${ }_{0}\left[d b \mathscr{S}\left(b_{N} ; i, j\right)\right]_{N+J-1+k}$ having mass vectors $e_{i} B^{J-1-k}$. That these rounds are possible and legal follows by induction on $k$.

We show that the graph $\mathscr{H}$ satisfies property (1). Recall that $\mathscr{G}_{1}$ is the graph resulting from the splittings in stages 1 and 2 . Each state of $\mathscr{G}_{1}$ is of the form ${ }_{0}[d]_{m}$, where $d$ is an $(m+1)$ path in $\mathscr{G}_{A}$ and $N-1 \leq m \leq L$, and $w\left({ }_{0}[d]_{m}\right)=S_{d_{m+1}, *} B^{L-m}$. 
If ${ }_{0}[d]_{m} \cap \Sigma_{\widetilde{A}} \neq \varnothing$, then $d=d_{1} d_{2} \cdots d_{m+1}$ is an $(m+1)$ path in $\mathscr{G}_{\widetilde{A}}$, so ${ }_{0}[d]_{m} \cap \Sigma_{\widetilde{A}}$ is the union of $(L+1)$ blocks ${ }_{0}[d \mathscr{S}]_{L} \cap \Sigma_{\tilde{A}}$, where $\mathscr{S}$ is the set of $(L-m)$ paths in $\mathscr{G}_{\widetilde{A}}$ with initial state a follower of state $d_{m+1}$. Now

$$
\begin{aligned}
w\left(_{0}[d]_{m}\right) & =S_{d_{m+1}, *} B^{L-m} \geq e_{\widetilde{\varphi}\left(d_{m+1}\right)} B^{L-m} \\
& =\Sigma\left[e_{t_{L-m}}: \widetilde{\varphi}\left(d_{m+1}\right) t_{1} t_{2} \cdots t_{L-m} \text { is a path in } \mathscr{G}_{B}\right] \\
& \geq \Sigma\left[e_{\widetilde{\varphi}\left(s_{L-m}\right)}: d_{m+1} s_{1} s_{2} \cdots s_{L-m} \text { is a path in } \mathscr{G}_{\widetilde{A}}\right],
\end{aligned}
$$

the last inequality follows because $\widetilde{\varphi}: \Sigma_{\widetilde{A}} \rightarrow \Sigma_{B}$ is right resolving.

Thus property (1) holds for $\mathscr{G}_{1}$. We now show that property (1) is preserved in the rounds of splitting in stages 3 and 4 , thus showing that (1) holds for $\mathscr{H}$ as well.

For convenience, we call a legal splitting that preserves property (1) $\tilde{\varphi}$ respecting.

At stage 3, each state in graph $\mathscr{G}_{1}$ of the form ${ }_{0}[b]_{N-1}, b \in \mathscr{B}$ is split into the states

$$
{ }_{0}\left[b \mathscr{S}\left(b_{N} ; i, j\right)\right]_{N+J-1}, \quad 1 \leq i \leq \beta, \quad 1 \leq j \leq S_{b_{N}, i} .
$$

To show that this splitting is $\widetilde{\varphi}$-respecting, we need to examine the daughter states having nonempty intersection with $\Sigma_{\tilde{A}}$. These are the states

$$
{ }_{0}\left[b \mathscr{S}\left(b_{N} ; \widetilde{\varphi}\left(b_{N}\right), 1\right)\right]_{N+J-1},
$$

where $b \in \mathscr{B}$ is an $N$ path in $\mathscr{G}_{\widetilde{A}}$. Abbreviate the displayed state by $s$. Now

$$
\begin{aligned}
w(s) & =e_{\widetilde{\varphi}\left(b_{N}\right)} B^{J-1}=\Sigma\left[e_{t_{J-1}}: \widetilde{\varphi}\left(b_{N}\right) t_{1} t_{2} \cdots t_{J-1} \text { is a path in } \mathscr{G}_{B}\right] \\
& \geq \Sigma\left[e_{\widetilde{\varphi}\left(s_{J-1}\right)}: b_{N} s_{1} s_{2} \cdots s_{J-1} \text { is a path in } \mathscr{G}_{\widetilde{A}}\right] \\
& =\Sigma\left[e_{\widetilde{\varphi}\left(s_{L+1}\right)}:{ }_{0}\left[s_{1} s_{2} \cdots s_{L+1}\right]_{L} \cap s \cap \Sigma_{\widetilde{A}} \neq \varnothing\right],
\end{aligned}
$$

the inequality holds because $\tilde{\varphi}: \Sigma_{\widetilde{A}} \rightarrow \Sigma_{B}$ is right resolving, and the last equality holds because $s \cap \Sigma_{\widetilde{A}}={ }_{0}[b]_{N-1} \cap \Sigma_{\widetilde{A}}$, which in turn follows from the fact that the set $\mathscr{S}\left(b_{N} ; \widetilde{\varphi}\left(b_{N}\right), 1\right)$ contains all the $J$ paths in $\mathscr{G}_{\widetilde{A}}$ following state $b_{N}$.

Thus, the round of splitting at stage 3 is $\widetilde{\varphi}$-respecting.

Similarly, the rounds of splitting at stage 4 are $\tilde{\varphi}$-respecting because

$$
{ }_{0}[d b]_{N-1+k} \cap \Sigma_{\widetilde{A}}={ }_{0}\left[d b \mathscr{S}\left(b_{N} ; \widetilde{\varphi}\left(b_{N}\right), 1\right)\right]_{N+J-1+k} \cap \Sigma_{\widetilde{A}},
$$

and

$$
w\left(0_{0}\left[d b \mathscr{S}\left(b_{N} ; \widetilde{\varphi}\left(b_{N}\right), 1\right)\right]_{N+J-1+k}\right)=e_{\widetilde{\varphi}\left(b_{N}\right)} B^{J-1-k},
$$

where $b \in \mathscr{B}, 0<k \leq J-1$, and $d b$ is an $(N+k)$-path in $\mathscr{G}_{\widetilde{A}}$. This shows that the graph $\mathscr{H}$ satisfies property (1).

One way to show (2), that $\mathscr{H}$ has only a finite number of fat cycles, is to show that each fat cycle in $\mathscr{H}$ is terminal: the only infinite fat path from any state on a fat cycle is the path winding forever around the cycle.

Let $x \in \Sigma_{\mathscr{Z}}$ be a point such that ${ }_{-\infty}(x)_{0}$ winds around a fat cycle $s_{0} s_{1} \cdots s_{p-1}$ and $x_{i}$ is fat for $i>0$. To show that the cycle $s_{0} s_{1} \cdots s_{p-1}$ is terminal, we must show that $x$ is actually a periodic point winding around the cycle $s_{0} s_{1} \cdots s_{p-1}$. 
Now $x$ avoids the lean states ${ }_{0}\left[d b \mathscr{S}\left(b_{N} ; i, j\right)\right]_{N+2 J-2}$, where $b \in \mathscr{B}$ and $d b$ is an $(M+J-1)$ path in $\mathscr{G}_{A}$. Since the union over all such $d, b, i, j$ of these states is

$$
\bigcup_{b \in \mathscr{B}} J_{-1}[b]_{N+J-2}
$$

the point $\alpha(x) \in \Sigma_{A}$ avoids the $N$ paths in $\mathscr{B}$, and so it is a periodic point in $\Sigma_{A}$ of period less than $J$. Since $\alpha$ is a conjugacy $x \in \Sigma_{\mathscr{H}}$ is a periodic point also, so $x$ must be some shift of $\left(s_{0} s_{1} \cdots s_{p-1}\right)^{\infty}$. This shows that any fat path leaving the cycle $s_{0} s_{1} \cdots s_{p-1}$ in $\mathscr{H}$ has finite length, which means the cycle is terminal.

We show property (3). Any fat cycle $\mathscr{C}$ in the graph $\mathscr{H}$ avoids the marker states ${ }_{0}\left[d b \mathscr{S}\left(b_{N} ; i, j\right)\right]_{N+J-1+|d|}$ so the states along $\mathscr{C}$ must be of the form ${ }_{0}[c]_{N+J-2}={ }_{0}[c]_{L}$, where $c$ is $j$ periodic for some $j<J$. Now

$$
w\left({ }_{0}\left[c_{1} c_{2} \cdots c_{L+1}\right]_{L}\right)=S_{c_{L+1}, *}
$$

this is property (4).

We now describe rounds of legal, $\tilde{\varphi}$-respecting splittings that are designed to isolate the fat cycles in $\mathscr{H}$. Because we will use this construction again, we set it off here as a claim.

(3.5) Claim. Suppose a graph $\mathscr{H}$ is obtained from $\mathscr{G}_{A}$ and $\mathscr{H}$ satisfies property (1): If $s$ is a state of $\mathscr{H}$ with $s \cap \Sigma_{\widetilde{A}} \neq \varnothing$, then $s \cap \Sigma_{\widetilde{A}}$ is a union of $(L+1)$ blocks in $\Sigma_{\widetilde{A}}$ and

$$
w(s) \geq \Sigma\left[e_{\widetilde{\varphi}\left(a_{L}\right)}:{ }_{0}\left[a_{0} a_{1} \cdots a_{L}\right]_{L} \cap s \cap \Sigma_{\widetilde{A}} \neq \varnothing\right] .
$$

Furthermore, assume that $s$ is a state of $\mathscr{H}$ with lean followers only. Then state $s$ can be split by a legal, $\widetilde{\varphi}$-respecting splitting entirely into lean states. Therefore, property (1) is preserved by this splitting.

Proof of Claim (3.5). Now the total mass of all the followers of state $s$ is $w(s) B$. A partition of the followers of state $s$ defining a merely legal splitting of $s$ entirely into lean states is easy to concoct: just partition the followers into

$$
\begin{aligned}
& w(s)_{1} \text { sets, each with total mass } B_{1, *}, \\
& w(s)_{2} \text { sets, each with total mass } B_{2, *}, \\
& \ldots \\
& w(s)_{\beta} \text { sets, each with total mass } B_{\beta, *} .
\end{aligned}
$$

As we now show, with a little more care we can ensure that such a partition defines a $\widetilde{\varphi}$-respecting splitting.

If $s \cap \Sigma_{\tilde{A}}=\varnothing$ there is nothing more to prove, so suppose $s \cap \Sigma_{\widetilde{A}} \neq \varnothing$. By assumption, $s \cap \Sigma_{\widetilde{A}}$ is a union of $(L+1)$ blocks in $\Sigma_{\tilde{A}}$. By property (1), any lean state of $\mathscr{H}$ can intersect $\Sigma_{\tilde{A}}$ in at most one $(L+1)$ block of $\mathscr{H}$. In particular, this is true of each follower of state $s$. Let

$$
{ }_{0}\left[a^{k}\right]_{L}={ }_{0}\left[a_{0}^{k} a_{1}^{k} \cdots a_{L}^{k}\right]_{L}, \quad 1 \leq k \leq K,
$$

be the $(L+1)$ blocks such that ${ }_{0}\left[a^{k}\right]_{L} \cap \Sigma_{\widetilde{A}} \cap s \neq \varnothing$.

We now show that one can partition the followers of state $s$ into sets with mass distributed as above, but with the added constraint that for each $1 \leq k \leq$ 
$K$, the set of states

$$
\mathscr{Q}_{k}=\left\{t: t \text { follows } s \text { in } \mathscr{H} \text { and } \varnothing \neq t \cap \Sigma_{\widetilde{A}} \subseteq 0\left[a_{1}^{k} a_{2}^{k} \cdots a_{L}^{k}\right]_{L-1} \cap \Sigma_{\widetilde{A}}\right\}
$$

be contained entirely in a single partition set. Because $\mathscr{H}$ satisfies property (1), $w(s) \geq \sum_{k=1}^{K} e_{\widetilde{\varphi}\left(a_{L}^{k}\right)}$, so we have a lower bound for the total mass of the followers of $s$ :

$$
w(s) B \geq \sum_{k=1}^{K} e_{\widetilde{\varphi}\left(a_{L}^{k}\right)} B .
$$

Because $\tilde{\varphi}: \Sigma_{\widetilde{A}} \rightarrow \Sigma_{B}$ is right resolving, we have

$$
\begin{aligned}
e_{\widetilde{\varphi}\left(a_{L}^{k}\right)} B & \geq \Sigma\left[e_{\widetilde{\varphi}(f)}: \text { state } f \text { follows state } a_{L}^{k} \text { in graph } \mathscr{G}_{\widetilde{A}}\right] \\
& =\Sigma\left[w(t): t \in \mathscr{Q}_{k}\right]=w\left(\mathscr{Q}_{k}\right),
\end{aligned}
$$

where the first equality holds because a follower $t$ of state $s$ in graph $\mathscr{H}$ is in $\mathscr{Q}_{k}$ iff

$$
t \cap \Sigma_{\widetilde{A}}={ }_{0}\left[a_{1}^{k} a_{2}^{k} \cdots a_{L}^{k} f\right]_{L} \cap \Sigma_{\widetilde{A}}
$$

for some follower $f$ of state $a_{L}^{k}$ in graph $\mathscr{G}_{\widetilde{A}}$. Thus we can augment each set $\mathscr{Q}_{K}$ by throwing in more followers of state $s$, to build a set $\mathscr{P}_{k} \supseteq \mathscr{Q}_{k}$ such that $w\left(\mathscr{P}_{k}\right)=e_{\widetilde{\varphi}\left(a_{L}^{k}\right)} B$, while the $\mathscr{P}_{k}$ 's remain, as the $\mathscr{Q}_{k}$ 's, pairwise disjoint. is

The total mass of the followers of state $s$ that are not elements of $\bigcup_{k=1}^{K} \mathscr{P}_{k}$

$$
w(s) B-\sum_{k=1}^{K} e_{\widetilde{\varphi}\left(a_{L}^{k}\right)} B,
$$

and these can be partitioned as before into $\left[w(s)-\sum_{k=1}^{K} e_{\widetilde{\varphi}\left(a_{L}^{k}\right)}\right]_{i}$ sets, each with total mass $B_{i, *}$, for $1 \leq i \leq \beta$.

If state $s$ is split according to this partition, and the daughter states are assigned elementary masses in the natural way, then the daughter state $s^{k}$ of $s$ corresponding to partition set $\mathscr{P}_{k}$ has

$$
s^{k} \cap \Sigma_{\widetilde{A}}={ }_{0}\left[a_{0}^{k} a_{1}^{k} \cdots a_{L}^{k}\right]_{L} \cap \Sigma_{\widetilde{A}},
$$

and $w\left(s^{k}\right)=e_{\widetilde{\varphi}\left(a_{L}^{k}\right)}$, so the splitting is $\tilde{\varphi}$-respecting. This completes the proof of the claim.

Recall that each fat cycle $\mathscr{C}$ in $\mathscr{H}$ is terminal: all fat paths in $\mathscr{H}$ leaving $\mathscr{C}$ have finite length. Using Claim (3.5) we can split using a round of $\tilde{\varphi}$-respecting splitting the terminal fat state of all maximal-length fat paths leaving the fat cycle $\mathscr{C}$, thereby shortening all maximal-length fat paths leaving $\mathscr{C}$ by length 1. Not only is this splitting $\tilde{\varphi}$-respecting (preserving property $(1)$ ), but it also preserves properties (2), (3), and (4) of the graph $\mathscr{H}$. By induction, we can continue shortening the fat paths leaving the fat cycle $\mathscr{C}$ until $\mathscr{C}$ is isolated. Doing this for all fat cycles, we reach our goal, graph $\mathscr{G}^{\prime}$ in which each fat cycle is isolated. This completes the proof of Lemma (3.4).

We now deal with the problem of splitting isolated fat cycles in $\mathscr{G}^{\prime}$ by rounds of $\tilde{\varphi}$-respecting splitting.

We can treat the problem of splitting an isolated fat cycle $\mathscr{C}$ in isolation from the rest of the graph $\mathscr{G}^{\prime}$. This is because any state $s$ of $\mathscr{G}^{\prime}$ immediately 
forwardly accessible from $\mathscr{C}$ is lean and hence cannot be further split by legal splitting; thus what follows state $s$ in $\mathscr{G}^{\prime}$ can have no bearing on how states along $\mathscr{C}$ can be legally split.

For this reason we regard the isolated fat cycle $\mathscr{C}$ as sitting in an isolated fat cycle graph comprising a single (simple) cycle $s_{0}^{\prime} s_{1}^{\prime} \cdots s_{p-1}^{\prime}$ together with the immediate followers of state $s_{i}^{\prime}$ (all lean except $s_{i+1}^{\prime}$ ), $0 \leq i \leq p-1$.

By property (3) of graph $\mathscr{G}^{\prime}$ given in the Marker Splitting Lemma (3.4) the sequence of state masses along a fat cycle $s_{1}^{\prime} s_{1}^{\prime} \cdots s_{p-1}^{\prime}$ in $\mathscr{G}^{\prime}$ is given by $w\left(s_{i}^{\prime}\right)=S_{s_{i}, *}$, where $s_{i}^{\prime}={ }_{0}\left[s_{i-L} s_{i-L+1} \cdots s_{i}\right]_{L}$ and $s_{0} s_{1} \cdots s_{p-1}$ is a cycle in the original graph $\mathscr{G}_{A}$. We can replace $S$ in the statement of the Marker Splitting Lemma (3.4) by $S B^{n}$ for any $n \geq 0$, to obtain a graph $\mathscr{G}^{\prime}$ in which any fat cycle $s_{0}^{\prime} s_{1}^{\prime} \cdots s_{p-1}^{\prime}$ has state masses given by $w\left(s_{i}^{\prime}\right)=S_{s_{i}, *} B^{n}$, where again $s_{0} s_{1} \cdots s_{p-1}$ is a cycle in the original graph $\mathscr{G}_{A}$.

The following lemma will serve to eliminate all the fat cycles from $\mathscr{G}^{\prime}$.

(3.6) Isolated Cycle Lemma. Assume that $A, B, R, S, \Sigma_{\widetilde{A}} \subseteq \Sigma_{A}, \Sigma_{\widetilde{B}} \subseteq \Sigma_{B}$, and $\widetilde{\varphi}: \Sigma_{\widetilde{A}} \rightarrow \Sigma_{\widetilde{B}}$ satisfy all the hypotheses of the Marker Splitting Lemma (3.4). In addition, assume that $\Sigma_{A} \stackrel{\text { per }}{\rightarrow} \Sigma_{B}$. Then there is an $N>0$ such that for all $n \geq N$, for all cycles $s_{0} s_{1} \cdots s_{p-1}$ in $\mathscr{G}_{A}$, an isolated fat cycle $s_{0}^{\prime} s_{1}^{\prime} \cdots s_{p-1}^{\prime}$ in $\mathscr{G}^{\prime}$ with $s_{1}^{\prime}={ }_{0}\left[s_{i-L} \cdots s_{i}\right]_{L}$ and $w\left(s_{i}^{\prime}\right)=S_{s_{i}, *} B^{n}$ can be split completely into lean states by rounds of legal, $\tilde{\varphi}$-respecting splitting.

To prove this lemma we study the class of graphs obtained by splitting isolated p-cycles: $p$-cycles of trees.

A $p$-cycle of trees is a union of $p$ disjoint directed trees (with edges directed away from the root and toward the leaves), where the roots $r_{0}, r_{1}, \ldots, r_{p-1}$ of the trees are connected in a directed cycle $r_{0} r_{1} \cdots r_{p-1}$.

Notice that an isolated fat cycle together with the states immediately accessible from it form a cycle of trees.

We define the phase of a node $s$ in a $p$-cycle of trees $\mathscr{T}$ to be $|w s| \bmod p$ where $r_{0} w s$ is any path in $\mathscr{T}$ from a fixed root $r_{0}$ to the state $s$. If a round of state splitting is applied to a cycle of tress $\mathscr{T}$ we obtain a new cycle of trees and we can consistently assign to each state in the new cycle of trees the phase of its ancestor.

We define a resolving covering graph of a graph $\mathscr{G}_{B}$ to be a directed graph $\mathscr{G}$ such that there is a map $\pi$ from the states of $\mathscr{G}$ to the states of $\mathscr{G}_{B}$ that is right resolving at each internal node of $\mathscr{G}$, where an internal node is a state of $\mathscr{G}$ having followers in $\mathscr{G}$. Further, for each internal node $s$ of $\mathscr{G}$, and each follower $t^{\prime}$ of $\pi(s)$ in $\mathscr{G}_{B}$, there is a follower $s^{\prime}$ of $s$ in $\mathscr{G}$ with $\pi\left(s^{\prime}\right)=t^{\prime}$.

If an isolated cycle $s_{0} s_{1} \cdots s_{p-1}$ is split by legal rounds of state splitting into a graph $\mathscr{T}$ with all lean states, then $\mathscr{T}$ is a cycle of trees that forms a resolving covering graph of $\mathscr{G}_{B}$, covering $\mathscr{G}_{B}$ with the mass map.

Since state splitting preserves phase in a cycle of trees, we have

$$
\Sigma[w(s): s \text { if an internal node of } \mathscr{T} \text { having phase } i]=w\left(s_{i}\right) .
$$

We have proved the only if part of the following observation.

(3.7) Observation. An isolated fat cycle $s_{0} s_{1} \cdots s_{p-1}$ can be completely split into lean states by rounds of legal splitting if and only if there is a $p$ cycle of 
trees $\mathscr{T}$ that is a resolving covering graph of $\mathscr{G}_{B}$ by state map $\pi$ such that

$$
\Sigma\left[e_{\pi(s)}: s \text { is an internal node of } \mathscr{T} \text { having phase } i\right]=w\left(s_{i}\right) .
$$

The proof of the if part is similar to the proof of Observation (3.9) below, or see [A2].

Observation (3.7) characterizes the isolated fat cycles that can be split into lean states by legal splittings. We will need more than this in order to split certain isolated cycles in $\mathscr{G}^{\prime}$ by $\widetilde{\varphi}$-respecting splittings. Observation (3.9) below meets this need. The isolated fat cycles of $\mathscr{G}^{\prime}$ fall into two types.

(3.8) Lemma. Let $\mathscr{G}^{\prime}$ be the graph produced by the Marker Splitting Lemma (3.4), and let $\mathscr{C}=s_{1}^{\prime} s_{1}^{\prime} \cdots s_{p-1}^{\prime}$ be an isolated fat cycle in $\mathscr{G}^{\prime}$. Then either each state along $\mathscr{C}$, regarded as a subset of $\Sigma_{A}$, is disjoint from $\Sigma_{\widetilde{A}}$, or each state along $\mathscr{C}$ intersects $\Sigma_{\widetilde{A}}$ in an $(L+1)$-block in $\Sigma_{\widetilde{A}}$.

Proof. Each state $s_{i}^{\prime}$ along an isolated fat cycle $\mathscr{C}$ in $\mathscr{G}^{\prime}$ is an $(L+1)$-block ${ }_{0}\left[s_{i} s_{i+1} \cdots s_{i+L}\right]_{L}$ in $\Sigma_{A}$, where $s_{0} s_{1} \cdots s_{p-1}$ is a $p$ cycle in $\mathscr{G}_{A}$. If $s_{0} s_{1} \cdots s_{p-1}$ is a $p$-cycle in $\mathscr{G}_{\widetilde{A}}$, then each $s_{i}^{\prime}$ intersects $\Sigma_{\widetilde{A}}$ in an $(L+1)$-block in $\Sigma_{\widetilde{A}}$. If $s_{0} s_{1} \cdots s_{p-1}$ is not a $p$-cycle in $\mathscr{G}_{\widetilde{A}}$ then at least one state $s_{i_{0}}$ along the $p$-cycle is not in $\mathscr{G}_{\widetilde{A}}$. Now since $L+1=N+J-1 \geq J-1 \geq p$, we have

$$
{ }_{0}\left[s_{i} s_{i+1} \cdots s_{i+L}\right]_{L} \subseteq \sigma^{-k}{ }_{0}\left[s_{i_{0}}\right]_{0}
$$

where $0 \leq k \leq L$, and $k \equiv i_{0}-i(\bmod p)$. But ${ }_{0}\left[s_{i_{0}}\right]_{0} \cap \Sigma_{\widetilde{A}}=\varnothing$.

If $\mathscr{C}=s_{0}^{\prime} s_{1}^{\prime} \cdots s_{p-1}^{\prime}$ is an isolated cycle in $\mathscr{G}^{\prime}$ with $s_{i}^{\prime} \cap \Sigma_{\widetilde{A}}=\varnothing, 0 \leq i \leq p-1$, then any legal splitting of the states along $\mathscr{C}$ is $\tilde{\varphi}$-respecting. We now consider the other possibility. Each state $s_{i}^{\prime}$ along $\mathscr{C}$ intersects $\Sigma_{\widetilde{A}}$ in an $(L+1)$-block ${ }_{0}\left[s_{i-L} s_{i-L+1} \cdots s_{i}\right]_{L}$ in $\Sigma_{\widetilde{A}}$.

(3.9) Observation. Let $\mathscr{C}=s_{0}^{\prime} s_{1}^{\prime} \cdots s_{p-1}^{\prime}$ be an isolated cycle in $\mathscr{G}^{\prime}$ with $s_{i}^{\prime} \cap$ $\Sigma_{\widetilde{A}} \neq \varnothing, 0 \leq i \leq p-1$, (so $s_{i}^{\prime} \cap \Sigma_{\widetilde{A}}={ }_{0}\left[s_{i-L} s_{i-L+1} \cdots s_{i}\right]_{L} \cap \Sigma_{\widetilde{A}}$ where $s_{0} s_{1} \cdots s_{p-1}$ is a $p$-cycle in $\mathscr{G}_{\widetilde{A}}$ with $\left.p<J\right)$. For $0 \leq i \leq p-1$, let $\tilde{f}_{i, 1}, \tilde{f}_{i, 2}, \ldots, \tilde{f}_{i, n_{i}}$ be the followers of $s_{i}$ in the graph $\mathscr{G}_{\widetilde{A}}$ distinct from the follower $s_{i+1}$, if such exist. The isolated fat cycle $\mathscr{C}$ can be completely split into lean states by rounds of $\tilde{\varphi}$-respecting splittings if there is a p-cycle of trees $\widetilde{\mathscr{T}}$ that is a resolving graph covering $\mathscr{G}_{B}$ by state map $\pi$ satisfying

(i) $\pi\left(r_{i}\right)=\widetilde{\varphi}\left(s_{i}\right)$, where state $r_{i}$ in $\widetilde{\mathscr{T}}$ is the root at phase $i, 0 \leq i \leq p-1$,

(ii) the tree in $\widetilde{\mathscr{T}}$ with root $r_{i}$ has a leaf at depth 1 with $\pi$ image $\tilde{\varphi}\left(\tilde{f}_{i, j}\right)$, for $1 \leq j \leq n_{i}$,

(iii) $w\left(s_{i}^{\prime}\right)=\Sigma\left[e_{\pi(s)}: s\right.$ is an internal node of $\widetilde{\mathscr{T}}$ having phase $\left.i\right]$.

Proof of (3.9). The proof is an induction on the maximum depth of an internal node of $\widetilde{\mathscr{T}}$. If this maximum depth is 0 , then the only internal nodes of $\widetilde{\mathscr{T}}$ are the root nodes $r_{0}, r_{1}, \ldots, r_{p-1}$, so by (i) and (iii), $w\left(s_{i}^{\prime}\right)=e_{\pi\left(r_{i}\right)}=e_{\widetilde{\varphi}\left(s_{i}\right)}$. Thus no splitting needs to be done to completely split $\mathscr{C}$ into lean states.

Now suppose that the maximum depth of an internal node of $\widetilde{\mathscr{T}}$ is $n>0$. 
Condition (iii) gives that for $0 \leq i \leq p-1$,

$$
\begin{gathered}
\Sigma\left[w(t): t \text { is a follower of } s_{i}^{\prime} \text { other than } s_{i+1}^{\prime}\right]=w\left(s_{i}^{\prime}\right) B-w\left(s_{i+1}^{\prime}\right) \\
=\Sigma\left[e_{\pi(s)} B: s \text { is an internal node of } \widetilde{\mathscr{T}} \text { having phase } i\right] \\
\quad-\Sigma\left[e_{\pi(s)}: s \text { is an internal node of } \widetilde{\mathscr{T}} \text { having phase } i+1\right] \\
=\Sigma\left[e_{\pi(s)}: s \text { is any node of } \widetilde{\mathscr{T}} \text { having phase } i+1\right] \\
\quad-\Sigma\left[e_{\pi(s)}: s \text { is an internal node of } \widetilde{\mathscr{T}} \text { having phase } i+1\right] \\
=\Sigma\left[e_{\pi(s)}: s \text { is a leaf of } \widetilde{\mathscr{T}} \text { having phase } i+1\right] .
\end{gathered}
$$

Thus the leaves of $\widetilde{\mathscr{T}}$ at phase $i+1$ can be put into 1-to-1 correspondence with the followers of state $s_{i}^{\prime}$ in $\mathscr{G}^{\prime}$ other than $s_{i+1}^{\prime}$ in such a way that $e_{\pi(s)}=w(t)$, where leaf $s$ in $\widetilde{\mathscr{T}}$ corresponds to follower $t$ of state $s_{i}^{\prime}$ in $\mathscr{G}^{\prime}$. By condition (ii), we can arrange this correspondence so that for $1 \leq j \leq n_{i}$, there is a leaf $l_{i, j}$ at depth 1 , phase $i+1$, in $\widetilde{\mathscr{T}}$ with $\pi\left(l_{i, j}\right)=\widetilde{\varphi}\left(\tilde{f}_{i, j}\right)$ corresponding to the follower $f_{i, j}$ of $s_{i}^{\prime}$ in $\mathscr{G}^{\prime}$ with

$$
f_{i, j} \cap \Sigma_{\tilde{A}}={ }_{0}\left[s_{i-L+1} s_{i-L+2} \cdots s_{i} \tilde{f}_{i, j}\right]_{L} \cap \Sigma_{\widetilde{A}}
$$

and therefore with $w\left(f_{i, j}\right)=e_{\widetilde{\varphi}\left(\tilde{f}_{i, j}\right)}$.

Having fixed this correspondence, we define a $\tilde{\varphi}$-respecting round of splitting of $\mathscr{G}^{\prime}$ as follows. For $0 \leq i \leq p-1$, let $s_{i, 1}, \ldots, s_{i, m_{i}}$ be the internal nodes of $\widetilde{\mathscr{T}}$ at phase $i$ and depth $n$, if there are any. Partition the followers of state $s_{i}^{\prime}$ in $\mathscr{G}^{\prime}$ into $m_{i}+1$ subsets, $\mathscr{P}_{i, 0}, \mathscr{P}_{i, 1}, \ldots, \mathscr{P}_{i, m_{i}}$, where for $1 \leq j \leq m_{i}$, the elements of $\mathscr{P}_{i, j}$ are those followers of state $s_{i}^{\prime}$ in $\mathscr{G}^{\prime}$ that correspond to the followers of state $s_{i, j}$ in $\widetilde{\mathscr{T}}$. This makes sense because all the followers of state $s_{i, j}$ in $\widetilde{\mathscr{T}}$ are leaves. The elements of $\mathscr{P}_{i, 0}$ are the remaining followers of state $s_{i}^{\prime}$ in $\mathscr{G}^{\prime}$ (corresponding to leaves of $\mathscr{\mathscr { T }}$ at depth at most $n$ together with state $\left.s_{i+1}^{\prime}\right)$.

If we split $s_{i}^{\prime}$ according to this partition into states $s_{i, j}^{\prime}$ corresponding to these sets $\mathscr{P}_{i, j}, 0 \leq j \leq m_{i}$, and assign masses

$$
w\left(s_{i, j}^{\prime}\right)=e_{\pi\left(s_{i, j}\right)}, \quad 1 \leq j \leq m_{i},
$$

and

$w\left(s_{i, 0}^{\prime}\right)=\Sigma\left[e_{\pi(s)}: s\right.$ is an internal node of $\widetilde{\mathscr{T}}$ having phase $i$ and depth $\left.<n\right]$,

then the splitting is legal. We show that the splitting is $\tilde{\varphi}$-respecting. The followers of $s_{i}^{\prime}$ in $\mathscr{G}^{\prime}$ having nonempty intersection with $\Sigma_{\widetilde{A}}$, namely $f_{i, j}$, $1 \leq j \leq n_{i}$, and $s_{i+1}^{\prime}$, are all elements of the partition set $\mathscr{P}_{i, 0}$, giving

$$
s_{i, 0}^{\prime} \cap \Sigma_{\widetilde{A}}=s_{i}^{\prime} \cap \Sigma_{\widetilde{A}}={ }_{0}\left[s_{i-L} s_{i-L+1} \cdots s_{i}\right]_{L} \cap \Sigma_{\widetilde{A}},
$$

and $s_{i, j}^{\prime} \cap \Sigma_{\tilde{A}}=\varnothing, 1 \leq j \leq m_{i}$. Now $w\left(s_{i, 0}^{\prime}\right) \geq e_{\pi\left(r_{i}\right)}=e_{\widetilde{\varphi}\left(s_{i}\right)}$, so the splitting is $\widetilde{\varphi}$-respecting.

After this round of splitting the cycle $s_{0,0}^{\prime} s_{1,0}^{\prime} s_{2,0}^{\prime} \cdots s_{p-1,0}^{\prime}$ is an isolated cycle in the new graph. The cycle of trees $\mathscr{T}^{\prime} \subset \widetilde{\mathscr{T}}$ defined by omitting from $\widetilde{\mathscr{T}}$ all leaves at depth $n+1$ is a resolving covering graph of $\mathscr{G}_{B}$ by state map 
$\pi$ (restricted to $\mathscr{T}^{\prime}$ ) satisfying properties (i), (ii), and (iii) for the new isolated cycle $s_{0,0}^{\prime} s_{1,0}^{\prime} \cdots s_{p-1,0}^{\prime}$. The maximum depth of an internal node of $\mathscr{T}^{\prime}$ is $n-1$, so by the inductive hypothesis, the new isolated cycle can be completely split into lean states by rounds of $\widetilde{\varphi}$-respecting splitting. This completes the proof of Observation (3.9).

Observations (3.7) and (3.9) demonstrate that an isolated fat cycle in $\mathscr{G}^{\prime}$ can be split by $\widetilde{\varphi}$-respecting splittings when a cycle of trees satisfying certain properties exists. The remainder of the proof of the Isolated Cycle Lemma (3.6) focuses on the task of constructing this cycle of trees. We organize the construction as a series of technical lemmas.

For $n>0$ and any cycle $\mathscr{C}=s_{0} s_{1} \cdots s_{p-1}$ in $\mathscr{G}_{A}$, and any graph homomorphism $\psi: \mathscr{C} \rightarrow \mathscr{G}_{B}$ (onto a $q$ cycle, where $q \mid p$ ), we define a resolving cycle of trees $\mathscr{T}(n, \mathscr{C}, \psi)$ covering $\mathscr{G}_{B}$ by state map $\pi$ as follows:

(i) $\mathscr{T}(n, \mathscr{C}, \psi)$ has a cycle of roots $r_{0} r_{1} \cdots r_{p-1}$ with $\pi\left(r_{i}\right)=\psi\left(s_{i}\right), 0 \leq$ $i \leq p-1$,

(ii) for each follower $g$ of $\psi\left(s_{i}\right)$ in $\mathscr{G}_{B}$ except $\psi\left(s_{i+1}\right)$, the tree in $\mathscr{T}(n, \mathscr{C}, \psi)$ with root $r_{i}$ has a complete resolving subtree of depth $n$ covering $\mathscr{G}_{B}$, whose root covers $g$ by state map $\pi$. Thus all the leaves of $\mathscr{T}(n, \mathscr{C}, \psi)$ are at depth $n+1$.

Similarly, for $n>0$ and any cycle $\mathscr{C}=s_{0} s_{1} \cdots s_{p-1}$ in $\mathscr{G}_{\widetilde{A}}$, define a resolving cycle of trees $\widetilde{\mathscr{T}}(n, \mathscr{C})$ covering $\mathscr{G}_{B}$ by state map $\pi$ as follows:

(i) $\widetilde{\mathscr{T}}(n, \mathscr{C})$ has a cycle of roots $r_{0} r_{1} \cdots r_{p-1}$ with $\pi\left(r_{i}\right)=\widetilde{\varphi}\left(s_{i}\right), 0 \leq i \leq$ $p-1$,

(ii) the tree in $\widetilde{\mathscr{T}}(n, \mathscr{C})$ with root $r_{i}$ has a leaf at depth 1 with $\pi$-image $\tilde{g}_{i, j}, 1 \leq j \leq \tilde{n}_{i}$, where $\tilde{g}_{i, 1}, \tilde{g}_{i, 2}, \ldots, \tilde{g}_{i, \tilde{n}_{i}}$ are the followers of state $\widetilde{\varphi}\left(s_{i}\right)$ distinct from $\widetilde{\varphi}\left(s_{i+1}\right)$ in the graph $\mathscr{G}_{\widetilde{B}}$ (if there are any),

(iii) for each follower $g$ of $\widetilde{\varphi}\left(s_{i}\right)$ in $\mathscr{G}_{B}$ that is not a state in $\mathscr{G}_{\widetilde{B}}$, the tree in $\widetilde{\mathscr{T}}(n, \mathscr{C})$ with root $r_{i}$ has a complete resolving subtree of depth $n$ whose root covers $g$ by state map $\pi$.

Thus all the leaves of $\widetilde{\mathscr{T}}(n, \mathscr{C})$ are at depth 1 or $n+1$.

For any path $w=w_{1} w_{2} \cdots w_{n}$ in the graph $\mathscr{G}_{B}$ define the vector $I(w) \in \mathbb{N}^{\beta}$ by $I(w)=e_{w_{1}} B^{n-1}$. For any state $t$ in $\mathscr{G}_{\widetilde{B}}$ denote

$$
u(t)=\Sigma\left[e_{f}: f \text { follows } t \text { in } \mathscr{G}_{B} \text { and } f \text { is not a state in } \mathscr{G}_{\widetilde{B}}\right] .
$$

Note that $u(t) \neq 0$ for each state $t$ of $\mathscr{G}_{\widetilde{B}}$ by the escape hypothesis on $\mathscr{G}_{\widetilde{B}} \subseteq \mathscr{G}_{B}$.

For any path $w=w_{1} w_{2} \cdots w_{n}$ in $\mathscr{G}_{\widetilde{B}}$ define the vector $\widetilde{I}(w) \in \mathbb{N}^{\beta}$ by

$$
\widetilde{I}(w)=e_{w_{n}}+u\left(w_{n-1}\right)+u\left(w_{n-2}\right) B+\cdots+u\left(w_{1}\right) B^{n-2} .
$$

For any $p$-cycle of trees that covers $\mathscr{G}_{B}$ by state map $\pi$, define the $p$-by- $\beta$ matrix $\mathscr{I}(\mathscr{T})$ by

$$
\mathscr{I}(\mathscr{T})_{i, *}=\Sigma\left[e_{\pi(s)}: s \text { is an internal state of } \mathscr{T} \text { at phase } i\right] .
$$

(3.10) Claim. If $s_{0} s_{1} \cdots s_{p-1}$ is a $p$ cycle in $\mathscr{G}_{A}$ and $\psi\left(s_{0} \cdots s_{p-1}\right)$ is a $q$ cycle in $\mathscr{G}_{B}$ where $q \mid p$, then the cycle of trees $\mathscr{T}\left(n, s_{0} s_{1} \cdots s_{p-1}, \psi\right)$ covering $\mathscr{G}_{B}$ by state map $\pi$ satisfies

$$
\mathscr{I}\left(\mathscr{T}\left(n, s_{0} s_{1} \cdots s_{p-1}, \psi\right)\right)_{i, *}=I\left(\pi\left(s_{i-n} s_{i-n+1} \cdots s_{i}\right)\right)=e_{\pi\left(s_{i-n}\right)} B^{n} .
$$


Similarly, if $s_{0} s_{1} \cdots s_{p-1}$ is a $p$ cycle in $\mathscr{G}_{\widetilde{A}}$, then $\widetilde{\mathscr{T}}\left(n, s_{0} s_{1} \cdots s_{p-1}\right)$ is the cycle of trees satisfying

$$
\mathscr{I}\left(\widetilde{\mathscr{T}}\left(n, s_{0} s_{1} \cdots s_{p-1}\right)\right)_{i, *}=\tilde{I}\left(\tilde{\varphi}\left(s_{i-n} s_{i-n+1} \cdots s_{i}\right)\right),
$$

where the indices are regarded modulo $p$.

The proof is a simple calculation.

For two vectors $\vec{u}, \vec{v} \in \mathbb{R}^{n}$ let $\measuredangle(\vec{u}, \vec{v})$ be the angle $\theta$ formed by $\vec{u}$ and $\vec{v}$, where $0 \leq \theta \leq \pi$.

(3.11) Claim. Let $\vec{l}>0$ be a left Perron eigenvector for $B$. For all $\varepsilon>$ 0 there exists $K>0$ such that for all paths $w$ in $\mathscr{G}_{\widetilde{B}}$ with $|w| \geq K$, $\measuredangle(\vec{l}, \tilde{I}(w))<\varepsilon$ and for all paths $w$ in $\mathscr{G}_{B}$ with $|w| \geq K, \measuredangle(\vec{l}, I(w))<\varepsilon$.

Proof. Given $\varepsilon>0$. By Perron-Frobenius theory [S, Theorem 1.2], there is $k_{0}>0$ such that for $k \geq k_{0}$, the angle formed by $\vec{l}$ and each row of $B^{k}$ is less than $\varepsilon / 2$. Let $M$ by the maximum of the Euclidean norms

$$
\|\tilde{I}(w)\|_{2}=\left\|e_{w_{k_{0}+1}}+u\left(w_{k_{0}}\right)+u\left(w_{k_{0}-1}\right) B+\cdots+u\left(w_{1}\right) B^{k_{0}-1}\right\|_{2}
$$

over all words $w$ of length $k_{0}+1$ in $\mathscr{G}_{\widetilde{B}}$. Choose $k_{1}>0$ such that for all $k \geq k_{1}$, and for $1 \leq i \leq \beta$,

$$
\left\|B_{i, *}^{k_{0}+k-1}\right\|_{2}>\frac{M}{\sin (\varepsilon / 2)} .
$$

Let $x$ be a path in $\mathscr{G}_{\widetilde{B}}$ with $|x| \geq k_{0}+k_{1}+1$. Express $x=v w$ where $|v|=k \geq k_{1}$ and $|w|=k_{0}+1$. Now

$$
\widetilde{I}(x)=\tilde{I}(w)+\left[u\left(v_{1}\right) B^{k-1}+u\left(v_{2}\right) B^{k-2}+\cdots+u\left(v_{k}\right)\right] B^{k_{0}} .
$$

Abbreviating the second term as $\vec{v}$, we have $\|\tilde{I}(w)\|_{2} \leq M$ and $\|\vec{v}\|_{2}>$ $M / \sin (\varepsilon / 2)$. Also $\measuredangle(\vec{l}, \tilde{I}(w))<\pi / 2$ and $\measuredangle(\vec{l}, \vec{v})<\varepsilon / 2$.

If $\theta=\measuredangle(\vec{l}, \widetilde{I}(x))$, then

$$
\begin{aligned}
\tan \theta & <\frac{M+\|\vec{v}\|_{2} \sin (\varepsilon / 2)}{\|\vec{v}\|_{2} \cos (\varepsilon / 2)}=\frac{M}{\|\vec{v}\|_{2} \cos (\varepsilon / 2)}+\tan (\varepsilon / 2) \\
& <\frac{\sin (\varepsilon / 2)}{\cos (\varepsilon / 2)}+\tan (\varepsilon / 2)=2 \tan (\varepsilon / 2)<\tan (\varepsilon)
\end{aligned}
$$

so $\theta<\varepsilon$. The proof that $\measuredangle(\vec{l}, I(w))<\varepsilon$ for long paths $w$ is easier and we omit it.

(3.12) Claim. For all $\varepsilon>0$, there exists $L>0$ such that for all $l \geq L$, for all states $s$ of $\mathscr{G}_{A}, \measuredangle\left(\vec{l}, w(s) B^{l}\right)<\varepsilon$. The proof follows from Perron-Frobenius theory $[\mathrm{S}]$.

Claims (3.13) and (3.14) give the base case of an inductive construction of cycles of trees whose existence by Observations (3.7) and (3.9), ensures that we can split isolate fat cycles in $\mathscr{G}^{\prime}$. Claims (3.15) and (3.16) will give the inductive step in the construction. We bring the strands of the construction together in Claim (3.17). 
(3.13) Claim. For all $\delta>0$, there is $L>0$ such that for all $l \geq L$, for all states $s$ in $\mathscr{G}_{A}$, for all paths $w$ in $\mathscr{G}_{\widetilde{B}}$, if $\widetilde{I}(w) \not \leq w(s) B^{l}$, then

$$
(1-\delta) w(s) B^{l}<\widetilde{I}(w) .
$$

Similarly, for all $\delta>0$, there is $L>0$ such that for all $l \geq L$, for all states $s$ in $\mathscr{G}_{A}$, for all paths $w$ in $\mathscr{G}_{B}$, if $I(w) \not z w(s) B^{l}$, then

$$
(1-\delta) w(s) B^{l}<I(w)
$$

Proof. The idea is that for large $l, \widetilde{I}(w)$ and $w(s) B^{l}$ are nearly parallel.

For

$$
0 \leq \varepsilon<\min _{1 \leq i \leq \beta} \sin ^{-1}\left(l_{i} /\|\vec{l}\|_{2}\right)
$$

and $1 \leq i, j \leq \beta$, define

$$
\rho_{i j}(\varepsilon)=\frac{\min \left\{x_{i}: x_{j}=1 \text { and } \measuredangle(\vec{l}, \vec{x}) \leq \varepsilon\right\}}{\max \left\{x_{i}: x_{j}=1 \text { and } \measuredangle(\vec{l}, \vec{x}) \leq \varepsilon\right\}} .
$$

Note that for $\varepsilon$ in the range of definition, the set $\left\{\vec{x}: x_{j}=1\right.$ and $\measuredangle(\vec{l}, \vec{x}) \leq$ $\varepsilon\}$ is a solid ellipsoid, As $\varepsilon \rightarrow 0$, the major diameter of this ellipsoid goes to zero while the point $\vec{l} / l_{j}$ remains in its interior. Thus $\rho_{i j}(\varepsilon) \rightarrow 1$ as $\varepsilon \rightarrow 0$. For

$$
0 \leq \varepsilon<\min _{1 \leq j \leq \beta} \sin ^{-1}\left(l_{j} /\|\vec{l}\|_{2}\right),
$$

the function $\rho(\varepsilon)=\min _{1 \leq i, j \leq \beta} \rho_{i j}(\varepsilon)$ is continuous and $\rho(0)=1$.

Given $\delta>0$, choose $\varepsilon>0$ such that $1-\delta<\rho(\varepsilon)$. For this $\varepsilon>0$, fix $K>0$ as in Claim (3.11). Now fix $L>0$ such that (i) for all states $s$ of $\mathscr{G}_{A}$, and for all $l \geq L, \measuredangle\left(\vec{l}, w(s) B^{l}\right)<\varepsilon$ (use Claim (3.12)), (ii) for all paths $w$ in $\mathscr{G}_{\widetilde{B}}$ with $|w|<K$, and for all states $s$ in $\mathscr{G}_{A}, \widetilde{I}(w) \leq w(s) B^{L}$.

Let $l \geq L$, let $s$ be a state in $\mathscr{G}_{A}$, and let $w$ be a path in $\mathscr{G}_{\widetilde{B}}$ such that $\widetilde{I}(w) \not \leq w(s) B^{l}$. For any state $t$ in $\mathscr{G}_{A}$ following $s$ along a path of length $l-L$, we have $\widetilde{I}(w) \not \leq w(t) B^{L}$. This and property (ii) give $K \leq|w|$. By Claim (3.11) and the choice of $K$, we have $\measuredangle(\vec{l}, \widetilde{I}(w))<\varepsilon$. Since $\widetilde{I}(w) \not z w(s) B^{l}$, there is an index $1 \leq j \leq \beta$ such that $\left[w(s) B^{l}\right]_{j}<\widetilde{I}(w)_{j}$. We need to show that $(1-\delta)\left[w(s) B^{l}\right]_{i}<\widetilde{I}(w)_{i}$ for $i \neq j$. Now $\widetilde{I}(w) \in\left\{\vec{x}: x_{j} \geq\left[w(s) B^{l}\right]_{j}\right.$ and $\measuredangle(\vec{l}, \vec{x}) \leq \varepsilon\}$.

Since the coordinate function $x_{i}$ increases in the direction of any vector $\vec{u}$ with $\measuredangle(\vec{l}, \vec{u}) \leq \varepsilon$, the minimum of $x_{i}$ over the above set (an infinite truncated cone) is achieved on its flat boundary face $\left\{\vec{x}: x_{j}=\left[w(s) B^{l}\right]_{j}\right.$ and $\measuredangle(\vec{l}, \vec{x}) \leq \varepsilon\}$. Hence

$$
\begin{aligned}
\tilde{I}(w)_{i} & \geq \min \left\{x_{i}: x_{j}=\left[w(s) B^{l}\right]_{j} \text { and } \measuredangle(\vec{l}, \vec{x}) \leq \varepsilon\right\} \\
& =\left[w(s) B^{l}\right]_{j} \min \left\{x_{i}: x_{j}=1 \text { and } \measuredangle(\vec{l}, \vec{x}) \leq \varepsilon\right\}
\end{aligned}
$$


Since $\measuredangle\left(\vec{l}, w(s) B^{l}\right)<\varepsilon$ (property (i)), we have

$$
\begin{aligned}
{\left[w(s) B^{l}\right]_{i} } & \leq \max \left\{x_{i}: x_{j}=\left[w(s) B^{l}\right]_{j} \text { and } \measuredangle(\vec{l}, \vec{x}) \leq \varepsilon\right\} \\
& =\left[w(s) B^{l}\right]_{j} \max \left\{x_{i}: x_{j}=1 \text { and } \measuredangle(\vec{l}, \vec{x}) \leq \varepsilon\right\}
\end{aligned}
$$

Thus

$$
\frac{\widetilde{I}(w)_{i}}{\left[w(s) B^{l}\right]_{i}} \geq \frac{\left[w(s) B^{l}\right]_{j}}{\left[w(s) B^{l}\right]_{j}} \rho_{i j}(\varepsilon)=\rho_{i j}(\varepsilon) \geq \rho(\varepsilon)>1-\delta .
$$

Thus $\widetilde{I}(w)>(1-\delta) w(s) B^{l}$. The statement regarding $I(w)$ is proved similarly.

(3.14) Claim. For all $\delta>0$, there exists $L>0$ such that for all $l \geq L$, for all cycles $\mathscr{C}=s_{0} s_{1} \cdots s_{p-1}$ in $\mathscr{G}_{\widetilde{A}}$, there is a resolving cycle of trees $\widetilde{\mathscr{T}}$ covering $\mathscr{G}_{B}$ by state map $\pi$ satisfying

(i) $\pi\left(r_{i}\right)=\widetilde{\varphi}\left(s_{i}\right), 0 \leq i \leq p-1$, where $r_{0} r_{1} \cdots r_{p-1}$ is the cycle in $\widetilde{\mathscr{T}}$,

(ii) the tree with root $r_{i}$ in $\widetilde{\mathscr{T}}$ has a leaf at depth 1 with $\pi$ image $\tilde{g}_{i, j}$, for each $1 \leq j \leq \tilde{n}_{i}$, where $\tilde{g}_{i, 1}, \ldots, \tilde{g}_{i, \tilde{n}_{i}}$ are the followers of state $\widetilde{\varphi}\left(s_{i}\right)$ in the graph $\mathscr{G}_{\widetilde{B}}$ distinct from $\widetilde{\varphi}\left(s_{i+1}\right)$,

(iii) $\mathscr{I}(\widetilde{\mathscr{T}})_{i, *} \leq w\left(s_{i}\right) B^{l}, 0 \leq i \leq p-1$,

(iv) there exists $i_{0}, 0 \leq i_{0} \leq p-1$, such that $(1-\delta) w\left(s_{i_{0}}\right) B^{l} \leq \mathscr{I}(\widetilde{\mathscr{T}})_{i_{0}, *}$.

Furthermore, for all $l \geq L$, for all graph homomorphisms $\psi: \mathscr{C} \rightarrow \mathscr{G}_{B}$ (mapping $\mathscr{C}$ onto a $q$ cycle in $\mathscr{G}_{B}$ with $q \mid p$ ) there is a resolving cycle of trees $\mathscr{T}$ covering $\mathscr{G}_{B}$ by state map $\pi$ satisfying

(i') $\pi\left(r_{i}\right)=\psi\left(s_{i}\right), 0 \leq i \leq p-1$, where $r_{0} r_{1} \cdots r_{p-1}$ is the cycle in $\mathscr{T}$,

(ii') $\mathscr{I}(\mathscr{T})_{i, *} \leq w\left(s_{i}\right) B^{l}, 0 \leq i \leq p-1$,

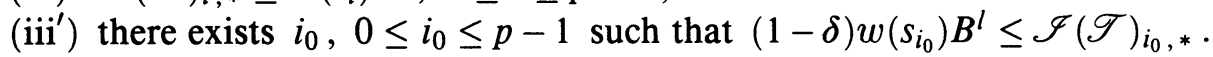

Proof. Given $\delta>0$. For this $\delta$, choose $L>0$ as in Claim (3.13), and fix $l \geq L$. Also fix a cycle $\mathscr{C}=s_{0} s_{1} \cdots s_{p-1}$ in $\mathscr{G}_{\widetilde{A}}$. Let $n$ be the largest integer such that

$$
\widetilde{I}\left(\tilde{\varphi}\left(s_{i-n} s_{i-n+1} \cdots s_{i}\right)\right) \leq w\left(s_{i}\right) B^{l}, \quad 0 \leq i \leq p-1 .
$$

We can assume $n \geq 1$ by increasing $L$ if necessary. By this choice of $n$, there is $i_{0}, 0 \leq i_{0} \leq p-1$, such that

$$
\widetilde{I}\left(\widetilde{\varphi}\left(s_{i_{0}-n-1} s_{i_{0}-n} \cdots s_{i_{0}}\right)\right) \not \leq w\left(s_{i_{0}}\right) B^{l} .
$$

Because $l \geq L$, by Claim (3.13) we have

$$
(1-\delta) w\left(s_{i_{0}}\right) B^{l}<\tilde{I}\left(\tilde{\varphi}\left(s_{i_{0}-n-1} s_{i_{0}-n} \cdots s_{i_{0}}\right)\right)
$$

so

$$
(1-\delta) w\left(s_{i_{0}}\right) B^{l}<\min \left\{\tilde{I}\left(\widetilde{\varphi}\left(s_{i_{0}-n-1} s_{i_{0}-n} \cdots s_{i_{0}}\right)\right), w\left(s_{i_{0}}\right) B^{l}\right\} .
$$

Abbreviate the componentwise minimum occurring on the right-hand side of the above inequality by $\vec{m}$.

We define the cycle of trees $\widetilde{\mathscr{T}}$ as follows: $\widetilde{\mathscr{T}}$ is a cycle of trees such that

$$
\widetilde{\mathscr{T}}\left(n, s_{0} s_{1} \cdots s_{p-1}\right) \subseteq \widetilde{\mathscr{T}} \subseteq \widetilde{\mathscr{T}}\left(n+1, s_{0} s_{1} \cdots s_{p-1}\right),
$$

where for $1 \leq j \leq \beta,\left[\vec{m}-\tilde{I}\left(\widetilde{\varphi}\left(s_{i_{0}-n} \cdots s_{i_{0}}\right)\right)\right]_{j}$ of the leaves of $\widetilde{\mathscr{T}}\left(n, s_{0} s_{1} \cdots s_{p-1}\right)$ having depth $n+1$, phase $i_{0}$, and $\pi$-image $j$, are defined to be internal 
nodes of $\widetilde{\mathscr{T}}$, and each is given $B_{j k}$ followers (leaves in $\widetilde{\mathscr{T}}$ ) with $\pi$-image $k$. That this is possible follows from the fact that the number of leaves of $\widetilde{\mathscr{T}}\left(n, s_{0} s_{1} \cdots s_{p-1}\right)$ having depth $n+1$, phase $i_{0}$, and $\pi$-image $j$ is

$$
\begin{aligned}
{\left[u\left(s_{i_{0}-n-1}\right) B^{n}\right]_{j} } & =\left[\tilde{I}\left(\tilde{\varphi}\left(s_{i_{0}-n-1} \cdots s_{i_{0}}\right)\right)-\tilde{I}\left(\tilde{\varphi}\left(s_{i_{0}-n} \cdots s_{i_{0}}\right)\right)\right]_{j} \\
& \geq\left[\vec{m}-\tilde{I}\left(\tilde{\varphi}\left(s_{i_{0}-n} \cdots s_{i_{0}}\right)\right)\right]_{j} .
\end{aligned}
$$

Thus

$$
\mathscr{I}(\widetilde{\mathscr{T}})_{i, *}=\left\{\begin{array}{lr}
\widetilde{I}\left(\widetilde{\varphi}\left(s_{i-n} \cdots s_{i}\right)\right. & \text { if } i \neq i_{0}, \\
\vec{m} & \text { if } i=i_{0},
\end{array}\right.
$$

so $\mathscr{I}(\widetilde{\mathscr{T}})_{i, *} \leq w\left(s_{i}\right) B^{l}, 0 \leq i \leq p-1$, and $(1-\delta) w\left(s_{i_{0}}\right) B^{l}<\mathscr{I}(\widetilde{\mathscr{T}})_{i_{0}, *}$, which are (iii) and (iv). Properties (i) and (ii) follow because $\widetilde{\mathscr{T}}$ is just $\widetilde{\mathscr{T}}\left(n, s_{0} s_{1} \cdots s_{p-1}\right)$ with some leaves grown from nodes at depth $n+1 \geq 2$.

A similar construction using $\mathscr{T}\left(n, s_{0} s_{1} \cdots s_{p-1}, \psi\right)$ for $\widetilde{\mathscr{T}}\left(n, s_{0} s_{1} \cdots s_{p-1}\right)$ and $I(w)$ in place of $\widetilde{I}(w)$ gives the tree $\mathscr{T}$ of the claim.

The trees $\widetilde{\mathscr{T}}$ and $\mathscr{T}$ of Claim (3.14) will serve as the bases for an inductive construction of cycles of trees satisfying the hypothesis of Observations (3.9) and (3.7) respectively. The induction will proceed by growing at successive steps, new leaves at successive phases.

Define $\vec{f}=(1,1, \ldots, 1)$ to be the row vector of $\beta 1$ 's.

(3.15) Claim. There exists $\delta>0$ and $L>0$ such that for all paths st of length 2 in $\mathscr{G}_{A}$ where $s$ has at least two followers (as is the case when $s$ is a state of $\left.\mathscr{G}_{\widetilde{A}}\right)$, if $l \geq L$ and $\vec{v} \in \mathbb{R}^{\beta}$ satisfies $(1-\delta) w(s) B^{l} \leq \vec{v}$, then $w(t) B^{l} \leq \vec{v} B-\vec{f}$.

Proof. Let $\vec{r}^{(B)}$ be a right Perron eigenvector of $B$ (for eigenvalue $\lambda$ ). Assume $\vec{r}(B)$ is scaled so that

$$
\left\|B^{n}-\lambda^{n} \vec{r}^{(B)} \vec{l}\right\|_{\infty}<\kappa \rho^{n}, \quad n>0,
$$

where $\kappa>0$ and $0<\rho<\lambda[\mathrm{S}$, Theorem 1.2]. It is easy to check that the column vector $\vec{r}(A)$ defined by $\vec{r}(A)=S \vec{r}(B)$ is a right Perron eigenvector for $A$. We have $w(s) \vec{r}^{(B)}=r_{s}^{(A)}$ for each state $s$ of $\mathscr{G}_{A}$. Fix $\delta>0$ such that $\nu=\min \left\{(1-\delta) \lambda r_{s}^{(A)}-r_{t}^{(A)}: s t\right.$ is a path of $\mathscr{G}_{A}$ and $s$ has followers distinct from $t\}>0$. For $l>0$ we have

$$
\begin{aligned}
(1-\delta) & w(s) B^{l+1}-w(t) B^{l} \\
= & (1-\delta) \lambda^{l+1} w(s) \vec{r}^{(B)} \vec{l}-\lambda^{l} w(t) \vec{r}^{(B)} \vec{l} \\
& +(1-\delta) w(s)\left[B^{l+1}-\lambda^{l+1} \vec{r}^{(B)} \vec{l}\right]-w(t)\left[B^{l}-\lambda^{l} \vec{r}^{(B)} \vec{l}\right] \\
& \geq(1-\delta) \lambda^{l+1} w(s) \vec{r}^{(B)} \vec{l}-\lambda^{l} w(t) \vec{r}^{(B)} \vec{l}-\mu \rho^{l} \vec{f},
\end{aligned}
$$

where $\mu>0$ is fixed and independent of $l, s$, and $t$; so

$$
\begin{aligned}
(1-\delta) w(s) B^{l+1}-w(t) B^{l} & \geq \lambda^{l}\left[(1-\delta) \lambda r_{s}^{(A)}-r_{t}^{(A)}\right] \vec{l}-\mu \rho^{l} \vec{f} \\
& \geq \lambda^{l} \nu \vec{l}-\mu \rho^{l} \vec{f}
\end{aligned}
$$


Fix $L>0$ such that for $l \geq L, \lambda^{l} \nu\left(\min _{1 \leq i \leq \beta} l_{i}\right)-\rho^{l} \mu>1$. For $l \geq L$, if $\vec{v} \in \mathbb{R}^{\beta}$ is such that $(1-\delta) w(s) B^{l} \leq \vec{v}$, then

$$
\begin{aligned}
\vec{v} B-w(t) B^{l} & \geq(1-\delta) w(s) B^{l+1}-w(t) B^{l} \\
& \geq \lambda^{l} v \vec{l}-\mu \rho^{l} \vec{f} \geq \vec{f},
\end{aligned}
$$

so $w(t) B^{l} \leq \vec{v} B-\vec{f}$ as claimed.

(3.16) Claim. There are $\delta>0$ and $L>0$ such that for all $l \geq L$, for all cycles $\mathscr{C}=s_{0} s_{1} \cdots s_{p-1}$ in $\mathscr{G}_{\widetilde{A}}$, if there is a resolving cycle of trees $\widetilde{\mathscr{T}}$ covering $\mathscr{G}_{B}$ by state map $\pi$ satisfying (i), (ii), (iii), and (iv) of Claim (3.14) (for this $\delta$ and $l \geq L)$, then there is a resolving cycle of trees $\widetilde{\mathscr{T}^{\prime}} \supseteq \widetilde{\mathscr{T}}$ covering $\mathscr{G}_{B}$, still satisfying (i), (ii), (iii), (iv) of Claim (3.14), while also satisfying

$$
w\left(s_{i_{0}+1}\right) B^{l}=\mathscr{I}(\widetilde{\mathscr{T}})_{i_{0}+1, *} .
$$

For the same $\delta>0$ and $L>0$, for all $l \geq L$, for all cycles $\mathscr{C}=s_{0} s_{1} \cdots s_{p-1}$ in $\mathscr{G}_{A}$, and all graph homomorphisms $\psi: \mathscr{C} \rightarrow \mathscr{G}_{B}$, if there is a resolving cycle of trees $\mathscr{T}$ covering $\mathscr{G}_{B}$ by state map $\pi$ satisfying $\left(\mathrm{i}^{\prime}\right)$, (ii'), and (iii') of Claim (3.14), then there is a resolving cycle of trees $\mathscr{T}^{\prime} \supseteq \mathscr{T}$ covering $\mathscr{G}_{B}$ still satisfying $\left(\mathrm{i}^{\prime}\right),\left(\mathrm{ii}^{\prime}\right)$, and $\left(\mathrm{iii}^{\prime}\right)$ of Claim (3.14) while also satisfying

$$
\rho w\left(s_{i_{0}+1}\right) B^{l} \leq \mathscr{I}\left(\mathscr{T}^{\prime}\right)_{i_{0}+1, *},
$$

where $\rho=\min _{1 \leq j \leq \beta} \mathscr{I}(\mathscr{T})_{i_{0}, j} /\left[w\left(s_{i_{0}}\right) B^{l}\right]_{j} \geq 1-\delta$, and even satisfying

$$
w\left(s_{i_{0}+1}\right) B^{l}=\mathscr{I}\left(\mathscr{T}^{\prime}\right)_{i_{0}+1, *}
$$

if state $s_{i_{0}}$ in $\mathscr{G}_{A}$ has followers distinct from $s_{i_{0}+1}$.

Proof. By Claim (3.15) we can fix $\delta>0$ and $L>0$ such that for all $l \geq L$, for all paths $s t$ of length 2 in $\mathscr{G}_{A}$ where state $s$ has followers distinct from $t$, if $\vec{v} \in \mathbb{R}^{\beta}$ is such that $(1-\delta) w(s) B^{l} \leq \vec{v}$, then

$$
w(t) B^{l} \leq \vec{v} B-\vec{f} .
$$

Assume $l \geq L, \mathscr{C}=s_{0} s_{1} \cdots s_{p-1}$ is a cycle in $\mathscr{G}_{\widetilde{A}}$, and $\widetilde{\mathscr{T}}$ is a resolving cycle of trees covering $\mathscr{G}_{B}$ by state map $\pi$ and satisfying (i), (ii), (iii), and (iv) for this $\delta$ and $l$. For the remainder of this proof assign mass $e_{\pi(s)}$ to each state $s$ of $\widetilde{\mathscr{T}}$.

By (iv), the total mass $\mathscr{I}(\mathscr{T})_{i_{0}, *}$ of the internal nodes of $\widetilde{\mathscr{T}}$ at phase $i_{0}$ satisfies $(1-\delta) w\left(s_{i_{0}}\right) B^{l} \leq \mathscr{I}(\mathscr{T})_{i_{0}, *}$. Now $\mathscr{I}(\mathscr{T})_{i_{0}, *} B$ is the total mass of all nodes of $\widetilde{\mathscr{T}}$ at phase $i_{0}+1$. Thus the total mass of all nodes of $\widetilde{\mathscr{T}}$ at phase $i_{0}+1$ excluding the $\tilde{n}_{i}$ leaves at depth 1 specified in (ii) satisfies

$$
\mathscr{I}(\widetilde{\mathscr{T}})_{i_{0}, *} B-\sum_{j=1}^{\tilde{n}_{i}} e_{\tilde{g}_{i, j}} \geq \mathscr{I}(\widetilde{\mathscr{T}})_{i_{0}, *} B-\vec{f} \geq w\left(s_{i_{0}+1}\right) B^{l},
$$

where the last inequality follows from $(1-\delta) w\left(s_{i_{0}}\right) B^{l} \leq \mathscr{I}(\mathscr{T})_{i_{0}, *}$ and the choice of $\delta>0$ given by Claim (3.15). Thus the total mass of the leaves of $\widetilde{\mathscr{T}}$ 
at phase $i_{0}+1$ excluding the $\tilde{n}_{i}$ leaves specified in (ii) is

$$
\begin{gathered}
\mathscr{I}(\widetilde{\mathscr{T}})_{i_{0}, *} B-\sum_{j=1}^{\tilde{n}_{i}} e_{\tilde{g}_{i, j}}-\mathscr{I}(\widetilde{\mathscr{T}})_{i_{0}+1, *} \\
\geq w\left(s_{\left.i_{0}+1\right)} B^{l}-\mathscr{I}(\widetilde{\mathscr{T}})_{i_{0}+1, *} \geq 0 .\right.
\end{gathered}
$$

Among these leaves, for each $1 \leq j \leq \beta$, choose $\left[w\left(s_{i_{0}+1}\right) B^{l}\right]_{j}-\mathscr{I}(\widetilde{\mathscr{T}})_{i_{0}+1, j}$ leaves, each having $\pi$ image $j$ and declare them to be internal nodes of $\widetilde{\mathscr{T}}^{\prime}$ giving each $B_{j k}$ children in $\widetilde{\mathscr{T}}^{\prime}$ with $\pi$-image $k, 1 \leq k \leq \beta$.

Now $\widetilde{\mathscr{T}^{\prime}}$ satisfies (i), (ii), (iii), (iv), and $w\left(s_{i_{0}+1}\right) B^{l}=\mathscr{I}\left(\widetilde{\mathscr{T}^{\prime}}\right)_{i_{0}+1, *}$, which is $(\mathrm{v})$.

Now assume $\mathscr{C}=s_{0} s_{1} \cdots s_{p-1}$ is a cycle in $\mathscr{G}_{A}$, and assume $\mathscr{T}$ is a resolving cycle of trees covering $\mathscr{G}_{B}$ by state map $\pi$ satisfying $\left(\mathrm{i}^{\prime}\right),\left(\mathrm{ii}^{\prime}\right)$, and (iii') of Claim (3.14) for the $\delta$ and $l \geq L$ specified above. As before, assign mass $e_{\pi(s)}$ to node $s$ of $\mathscr{T}$. The total mass of all nodes of $\mathscr{T}$ at phase $i_{0}+1$ is $\mathscr{I}(\mathscr{T})_{i_{0}, *} B$. Setting $\vec{m}=\min \left\{w\left(s_{i_{0}+1}\right) B^{l}, \mathscr{I}(\mathscr{T})_{i_{0}, *} B\right\}$ and arguing as in the case of $\widetilde{\mathscr{T}}$, we can declare enough leaves of $\mathscr{T}$ at phase $i_{0}+1$ to be internal nodes of $\mathscr{T}^{\prime}$ to bring $\mathscr{I}\left(\mathscr{T}^{\prime}\right)_{i_{0}+1, *}$ up to equal $\vec{m}$. Setting

$$
\rho=\min _{1 \leq j \leq \beta} \mathscr{I}(\mathscr{T})_{i_{0} j} /\left[w\left(s_{i_{0}}\right) B^{l}\right]_{j},
$$

we have $\rho w\left(s_{i_{0}+1}\right) B^{l} \leq \rho w\left(s_{i_{0}}\right) B^{l+1} \leq \mathscr{F}(\mathscr{T})_{i_{0}, *} B$, where by (iii'), $1-\delta \leq \rho$. So $\rho w\left(s_{i_{0}+1}\right) B^{l} \leq \vec{m}=\mathscr{I}\left(\mathscr{T}^{\prime}\right)_{i_{0}+1}$. This is $\left(\mathrm{iv}^{\prime}\right)$.

If state $s_{i_{0}}$ has followers in $\mathscr{G}_{A}$ other than $s_{i_{0}+1}$, it follows from Claim (3.15), and $(1-\delta) w\left(s_{i_{0}}\right) B^{l} \leq \mathscr{I}(\mathscr{T})_{i_{0}, *}$, and the choice of $\delta$ and $l \geq L$ that $w\left(s_{i_{0}+1}\right) B^{l} \leq \mathscr{I}(\mathscr{T})_{i_{0}, *} B$, so $\mathscr{I}\left(\mathscr{T}^{\prime}\right)_{i_{0}+1, *}=\vec{m}=w\left(s_{i_{0}+1}\right) B^{l}$ in this case. This is $\left(\mathbf{v}^{\prime}\right)$.

(3.17) Claim. There is $L>0$ such that for all $l \geq L$, for all cycles $\mathscr{C}=$ $s_{0} s_{1} \cdots s_{p-1}$ in $\mathscr{G}_{\widetilde{A}}$, there is a resolving cycle of tress $\widetilde{\mathscr{T}}$ covering $\mathscr{G}_{B}$ by state map $\pi$ satisfying

(i) $\pi\left(r_{i}\right)=\tilde{\varphi}\left(s_{i}\right), 0 \leq i \leq p-1$, where $r_{0} r_{1} \cdots r_{p-1}$ is the cycle in $\mathscr{T}$,

(ii) the tree in $\widetilde{\mathscr{T}}$ with root $r_{i}$ has a leaf at depth 1 with $\pi$ image $\tilde{g}_{i, j}$ for each $1 \leq j \leq \tilde{n}_{i}$, where $\tilde{g}_{i, 1}, \ldots, \tilde{g}_{i, \tilde{n}_{i}}$ are the followers of state $\widetilde{\varphi}\left(s_{i}\right)$ in the graph $\mathscr{G}_{\widetilde{B}}$ that are distinct from $\widetilde{\varphi}\left(s_{i+1}\right)$,

(iii) $w\left(s_{i}\right) B^{l}=\mathscr{I}(\widetilde{\mathscr{T}})_{i, *}, 0 \leq i \leq p-1$.

Furthermore, if $\Sigma_{A} \stackrel{\text { per }}{\rightarrow} \Sigma_{B}$, then for all $l \geq L$, for all cycles $\mathscr{C}=s_{0} s_{1} \cdots s_{p-1}$ in $\mathscr{G}_{A}$, there is a resolving $p$-cycle of trees $\mathscr{T}$ covering $\mathscr{G}_{B}$ by state map $\pi$ satisfying

(iii') $w\left(s_{i}\right) B^{l}=\mathscr{I}(\mathscr{T})_{i, *}, 0 \leq i \leq p-1$.

Proof. Let $\delta>0$ and $L_{1}>0$ satisfy the conditions on $\delta$ and $L$ specified in the statement of Claim (3.16). For this $\delta$, choose $L_{2}>0$ satisfying the conditions on $L$ specified in Claim (3.14). Set $L_{3}=\max \left\{L_{1}, L_{2}\right\}$.

Let $l \geq L_{3}$ and let $\mathscr{C}=s_{0} s_{1} \cdots s_{p-1}$ be any cycle in $\mathscr{G}_{\widetilde{A}}$. By Claim (3.14), there is a resolving cycle of trees $\widetilde{\mathscr{T}}^{(0)}$ covering $\mathscr{G}_{B}$ by state map $\pi$ satisfying 
conditions (i), (ii), (iii), and (iv) of Claim (3.14). By $p$ successive applications of Claim (3.16), there are cycles of trees $\widetilde{\mathscr{T}}^{(0)} \subseteq \widetilde{\mathscr{T}}^{(1)} \subseteq \cdots \subseteq \widetilde{\mathscr{T}}^{(p)}$ all satisfying (i), (ii), and (iii) of Claim (3.14); furthermore, for $1 \leq k \leq p, \widetilde{\mathscr{T}}^{(k)}$ satisfies

$$
w\left(s_{i_{0}+j}\right) B^{l}=\mathscr{I}\left(\widetilde{\mathscr{T}}^{(k)}\right)_{i_{0}+j, *}, \quad 1 \leq j \leq k .
$$

This follows directly from Claim (3.16) for $k=1$. For $k>1$, at the $k$ th application of Claim (3.16),

$$
(1-\delta) w\left(s_{i_{0}+k-1}\right) B^{l} \leq w\left(s_{i_{0}+k-1}\right) B^{l}=\mathscr{I}\left(\widetilde{\mathscr{T}}^{(k-1)}\right)_{i_{0}+k-1, *},
$$

which is condition (iv) of (3.14) (with $i_{0}+k-1$ playing the role of $i_{0}$ ), so Claim (3.16) gives $\widetilde{\mathscr{T}}^{(k)}$ as described. Now $\widetilde{\mathscr{T}}=\widetilde{\mathscr{T}}^{(p)}$ satisfies properties (i), (ii), and (iii) of the present claim.

Now let $\mathscr{C}=s_{0} s_{1} \cdots s_{p-1}$ be any cycle in $\mathscr{G}_{A}$. Because $\Sigma_{A} \stackrel{\text { per }}{\rightarrow} \Sigma_{B}$, there is a graph homomorphism $\psi: \mathscr{C} \rightarrow \mathscr{G}_{B}$. By Claim (3.14) there is a resolving cycle of trees $\mathscr{T}^{(0)}$ covering $\mathscr{G}_{B}$ by state map $\pi$ satisfying conditions $\left(\mathrm{i}^{\prime}\right),\left(\mathrm{ii}^{\prime}\right)$, and (iii') of Claim (3.14).

By the irreducibility of $\mathscr{G}_{A}$, there is a state on $\mathscr{C}$ with at least two followers; we let $s_{i_{0}+k_{0}}$ be the first such state following $s_{i_{0}-1}$ on $\mathscr{C}$. Note $0 \leq k_{0} \leq$ $p-1$. By $k_{0}$ successive applications of Claim (3.16), there are cycles of trees $\mathscr{T}^{(0)} \subseteq \mathscr{T}^{(1)} \subseteq \cdots \subseteq \mathscr{T}^{\left(k_{0}\right)}$ satisfying conditions $\left(\mathrm{i}^{\prime}\right)$ and (ii') of Claim (3.14); furthermore, for $1 \leq k \leq k_{0}, \mathscr{T}^{(k)}$ satisfies

$$
\rho w\left(s_{i_{0}+j}\right) B^{l} \leq \mathscr{I}\left(\mathscr{T}^{(k)}\right)_{i_{0}+j, *}, \quad 1 \leq j \leq k,
$$

where $1-\delta \leq \rho$. In particular, $(1-\delta) w\left(s_{i_{0}+k_{0}}\right) B^{l} \leq \mathscr{I}\left(\mathscr{T}^{\left(k_{0}\right)}\right)_{i_{0}+k_{0}, *}$.

Since state $s_{i_{0}+k_{0}}$ has at least two followers, we can apply Claim (3.16) once again to produce $\mathscr{T}^{\left(k_{0}+1\right)}$ containing $\mathscr{T}^{\left(k_{0}\right)}$ satisfying $w\left(s_{i_{0}+k_{0}+1}\right) B^{l}=$ $\mathscr{I}\left(\mathscr{T}^{\left(k_{0}+1\right)}\right)_{i_{0}+k_{0}+1, *}$. Now we apply Claim (3.16) $p-1$ more times to produce cycles of trees $\mathscr{T}^{\left(k_{0}+1\right)} \subseteq \mathscr{T}^{\left(k_{0}+2\right)} \subseteq \cdots \subseteq \mathscr{T}^{\left(k_{0}+p\right)}$ all satisfying (i') and (ii') of Claim (3.14); furthermore, for $1 \leq k \leq p, \mathscr{T}^{\left(k_{0}+k\right)}$ satisfies $w\left(s_{i_{0}+k_{0}+j}\right) B^{l}=\mathscr{I}\left(\mathscr{T}^{\left(k_{0}+k\right)}\right)_{i_{0}+k_{0}+j, *}, \quad 1 \leq j \leq k$. We show this as follows. Just before the $k$ th of these $p-1$ applications of Claim (3.16), we have $\rho=1$ (by inductive hypothesis), so conclusion (iv') of Claim (3.16) becomes

$$
w\left(s_{i_{0}+k_{0}+1+k}\right) B^{l} \leq \mathscr{I}\left(\mathscr{T}^{\left(k_{0}+1+k\right)}\right)_{i_{0}+k_{0}+1+k, *},
$$

while property $\left(\mathrm{ii}^{\prime}\right)$ of Claim (3.14) is preserved, giving

$$
w\left(s_{i_{0}+k_{0}+1+k}\right) B^{l}=\mathscr{J}\left(\mathscr{T}^{\left(k_{0}+1+k\right)}\right)_{i_{0}+k_{0}+1+k, *}
$$

as asserted. Now $\mathscr{T}=\mathscr{T}^{\left(k_{0}+p\right)}$ satisfies property (iii') of the present claim.

Using Observations (3.7), (3.9), and Claim (3.17), we can prove the Isolated Cycle Lemma (3.6).

Proof of (3.6). Let $N>0$ satisfy the conditions on $L$ specified in the statement of Claim (3.17). Let $\mathscr{C}=s_{0}^{\prime} s_{1}^{\prime} \cdots s_{p-1}^{\prime}$ be an isolated fat cycle in $\mathscr{G}^{\prime}$. Now $s_{i}^{\prime}={ }_{0}\left[s_{i-L} \cdots s_{i}\right]_{L}$ for some $p$ cycle $s_{0} s_{1} \cdots s_{p-1}$ in $\mathscr{G}_{A}$. By Lemma (3.8), either $s_{i}^{\prime} \cap \Sigma_{\widetilde{A}}=\varnothing, 0 \leq i \leq p-1$, or $s_{i}^{\prime} \cap \Sigma_{\widetilde{A}}$ is an $(L+1)$-block ${ }_{0}\left[s_{i-L} \cdots s_{i}\right]_{L} \cap \Sigma_{\widetilde{A}}$ in $\Sigma_{\widetilde{A}}, 0 \leq i \leq p-1$. We treat the case of nonempty intersection first. 
As in the statement of Observation (3.9), let $\tilde{f}_{i, 1}, \ldots, \tilde{f}_{i, n_{i}}$ be the followers of state $s_{i}$ in the graph $\mathscr{G}_{\widetilde{A}}$ distinct from the follower $s_{i+1}$, if there are any. Let $l \geq N$. For this $l$, let $\widetilde{\mathscr{T}}$ be a $p$-cycle of trees covering $\mathscr{G}_{B}$ by state map $\pi$ whose existence is asserted by Claim (3.17). We show that $\widetilde{\mathscr{T}}$ satisfies the hypothesis of Observation (3.9). $\widetilde{\mathscr{T}}$ satisfies property (i) of Observation (3.9): $\pi\left(r_{i}\right)=\widetilde{\varphi}\left(s_{i}\right), 0 \leq i \leq p-1$, where $r_{0} r_{1} \cdots r_{p-1}$ is the cycle in $\widetilde{\mathscr{T}}$, because this is just property (i) of Claim (3.17). Also $\widetilde{\mathscr{T}}$ satisfies property (ii) of Observation (3.9):

The tree in $\widetilde{\mathscr{T}}$ with root $r_{i}$ has a leaf at depth 1 with $\pi$-image $\tilde{\varphi}\left(\tilde{f}_{i, j}\right), 1 \leq j \leq n_{i}$,

because $\widetilde{\mathscr{T}}$ satisfies property (ii) of Claim (3.17):

The tree in $\widetilde{\mathscr{T}}$ with root $r_{i}$ has a leaf at depth 1 with $\pi$-image $\tilde{g}_{i, j}$ for each $1 \leq j \leq \tilde{n}_{i}$, where $\tilde{g}_{i, 1}, \ldots, \tilde{g}_{i, \tilde{n}_{i}}$ are the followers of state $\widetilde{\varphi}\left(s_{i}\right)$ in the graph $\mathscr{G}_{\widetilde{B}}$ that are distinct from $\widetilde{\varphi}\left(s_{i+1}\right)$,

and each of the states $\tilde{\varphi}\left(\tilde{f}_{i, j}\right)$ is among $\tilde{g}_{i, 1}, \ldots, \tilde{g}_{i, \tilde{n}_{i}}$. Finally $\widetilde{\mathscr{T}}$ satisfies property (iii) of Observation (3.9): $w\left(s_{i}^{\prime}\right)=\mathscr{I}(\widetilde{\mathscr{T}})_{i, *}, 0 \leq i \leq p-1$, when $w\left(s_{i}^{\prime}\right)=S_{s_{i}, *} B^{l}=w\left(s_{i}\right) B^{l}, 0 \leq i \leq p-1$, because this is property (iii) of Claim (3.17). So by Observation (3.9), the isolated fat cycle $s_{0}^{\prime} s_{1}^{\prime} \cdots s_{p-1}^{\prime}$ in $\mathscr{G}^{\prime}$ with masses given by $w\left(s_{i}^{\prime}\right)=S_{s_{i}, *} B^{l}$ can be split into lean states by rounds of legal, $\widetilde{\varphi}$-respecting splittings.

Now we treat the case where $s_{i}^{\prime} \cap \Sigma_{\widetilde{A}}=\varnothing, 0 \leq i \leq p-1$. Let $l \geq N$ and let $\mathscr{T}$ be the cycle of trees covering $\mathscr{G}_{B}$ by state map $\pi$ satisfying $w\left(s_{i}\right) B^{l}=$ $\mathscr{I}(\mathscr{T})_{i, *}, 0 \leq i \leq p-1$, whose existence is asserted by Claim (3.17). Now $\mathscr{T}$ satisfies the hypothesis of Observation (3.7) (when $w\left(s_{i}^{\prime}\right)=S_{s_{i}, *} B^{l}$ ), so the isolated fat cycle $s_{0}^{\prime} s_{1}^{\prime} \cdots s_{p-1}^{\prime}$ in $\mathscr{G}^{\prime}$ can be split by legal (and vacuously $\tilde{\varphi}$ respecting) rounds of splitting into lean states. This completes the proof of the Isolated Cycle Lemma (3.6).

We now complete the proof of Theorem (1.1).

Recall that by Lemma (3.1) we can assume without loss of generality that $A, B, R, S, \Sigma_{\widetilde{A}} \subseteq \Sigma_{A}, \Sigma_{\widetilde{B}} \subseteq \Sigma_{B}$, and $\widetilde{\varphi}: \Sigma_{\widetilde{A}} \rightarrow \Sigma_{\widetilde{B}}$ satisfy all the hypotheses of the Marker Splitting Lemma (3.4). In addition we are assuming that $\Sigma_{A} \stackrel{\text { per }}{\rightarrow} \Sigma_{B}$.

Let $N>0$ be as specified in the Isolated Cycle Lemma (3.6). Fix any $n \geq$ $N$. Replace the matrix $S$ by the matrix $S B^{n}$ in the statement of the Marker Splitting Lemma (3.4) to obtain a graph $\mathscr{G}^{\prime}$ in which any fat cycle $s_{0}^{\prime} s_{1}^{\prime} \cdots s_{p-1}^{\prime}$ has state masses given by $w\left(s_{i}^{\prime}\right)=S_{s_{i}, *} B^{n}$, where $s_{i}^{\prime}={ }_{0}\left[s_{i-L} \cdots s_{i}\right]_{L}$, and $s_{0} s_{1} \cdots s_{p-1}$ is a $p$ cycle in $\mathscr{G}_{A}$.

By the Isolated Cycle Lemma (3.6) and the choice of $n \geq N$, the isolated fat cycle $s_{0}^{\prime} s_{1}^{\prime} \cdots s_{p-1}^{\prime}$ in $\mathscr{G}^{\prime}$ can be split by rounds of $\tilde{\varphi}$-respecting splittings entirely into lean states.

Let $\mathscr{G}^{\prime \prime}$ be a graph obtained from $\mathscr{G}^{\prime}$ by splitting all the isolated fat cycles in $\mathscr{G}^{\prime}$ entirely into lean states by $\tilde{\varphi}$-respecting splittings. Because $\mathscr{G}^{\prime \prime}$ has no fat cycles, all remaining fat paths in $\mathscr{G}^{\prime \prime}$ have finite length. By Claim (3.5), the terminal fat state of any maximal length fat path in $\mathscr{G}^{\prime \prime}$ can be split by a legal, 
$\widetilde{\varphi}$-respecting splitting entirely into lean states, thereby shortening the maximal length fat path by length 1 . Iteratively eating up fat paths in this way, we can split all fat states in $\mathscr{G}^{\prime \prime}$ into lean states in at most $M$ rounds of $\tilde{\varphi}$-respecting splittings, where $M$ is the length of the longest fat path in $\mathscr{G}^{\prime \prime}$.

Denote the resulting graph by $\mathscr{G}_{A^{\prime}}$, where $A^{\prime}$ is its transition matrix. Because the graph $\mathscr{G}_{A^{\prime}}$ is obtained from $\mathscr{G}^{\prime}$ by rounds of $\tilde{\varphi}$-respecting splittings, $\mathscr{G}_{A^{\prime}}$, like $\mathscr{G}^{\prime}$, satisfies property (1) of the Marker Splitting Lemma (3.4):

If $s$ is a state of $\mathscr{G}_{A^{\prime}}$ and $s \cap \Sigma_{\widetilde{A}} \neq \varnothing$ then $s \cap \Sigma_{\widetilde{A}}$ is a union of $(L+1)$-blocks in $\Sigma_{\widetilde{A}}$ and

$$
w(s) \geq \Sigma\left[e_{\widetilde{\varphi}\left(s_{L}\right)}:{ }_{0}\left[s_{0} s_{1} \cdots s_{L}\right]_{L} \cap s \cap \Sigma_{\widetilde{A}} \neq \varnothing\right] .
$$

Together with the fact that all states of $\mathscr{G}_{A^{\prime}}$ are lean, this gives that each state $s$ of $\mathscr{G}_{A^{\prime}}$ intersects $\Sigma_{\widetilde{A}}$ in at most one $(L+1)$-block in $\Sigma_{\widetilde{A}}$. If $s \cap \Sigma_{\widetilde{A}} \neq \varnothing$, then

$$
s \cap \Sigma_{\widetilde{A}}={ }_{0}\left[s_{0} s_{1} \cdots s_{L}\right]_{L} \cap \Sigma_{\widetilde{A}},
$$

where $s_{0} s_{1} \cdots s_{L}$ is an $(L+1)$-path in $\mathscr{G}_{\widetilde{A}}$ and $w(s)=e_{\widetilde{\varphi}\left(s_{L}\right)}$. Let $\mu: \Sigma_{A^{\prime}} \rightarrow \Sigma_{B}$ be the right resolving 1-block mass map defined by the state map $\mu(s)=t$ where $w(s)=e_{t}$. If $\alpha: \Sigma_{A^{\prime}} \rightarrow \Sigma_{A}$ is the ancestor map, we have for each state $s$ of $\mathscr{G}_{A^{\prime}}$ with $s \cap \Sigma_{\widetilde{A}} \neq \varnothing, \mu(s)=\widetilde{\varphi}\left(s_{L}\right)$ and $\alpha(s)=s_{0}$, where

$$
s \cap \Sigma_{\widetilde{A}}={ }_{0}\left[s_{0} s_{1} \cdots s_{L}\right]_{L} \cap \Sigma_{\tilde{A}} .
$$

Thus

$$
\left.\left(\mu \circ \alpha^{-1}\right)\right|_{\Sigma_{\tilde{A}}}=\tilde{\varphi} \circ \sigma^{L} .
$$

So $\mu \circ \alpha^{-1}: \Sigma_{A} \rightarrow \Sigma_{B}$ is an extension of $\widetilde{\varphi} \circ \sigma^{L}$. If we define $\varphi: \Sigma_{A} \rightarrow \Sigma_{B}$ by $\varphi=\mu \circ \alpha^{-1}$, then $\varphi \circ \sigma^{-L}$ is an extension of $\tilde{\varphi}: \Sigma_{\tilde{A}} \rightarrow \Sigma_{B}$.

Now we can apply [A1] to conclude that there is a 1-to-1 almost everywhere right-closing map $\widehat{\varphi}: \Sigma_{A} \rightarrow \Sigma_{B}$. The construction in [A1] lowers the degree of a given map $\varphi: \Sigma_{A} \rightarrow \Sigma_{B}$ by altering the image of $x \in \Sigma_{A}$ between certain marker blocks in $x$ (these are preimages of resolving blocks). With some care, we can choose these marker blocks to be outside of $\Sigma_{\widetilde{A}}$, thus modifying the construction in [A1] to ensure that $\widehat{\varphi}$, like $\varphi$, is an extension of $\widetilde{\varphi} \circ \sigma^{L}$.

This completes the construction of $\varphi: \Sigma_{A} \rightarrow \Sigma_{B}$ and the proof of Theorem (1.1).

\section{THE SOFIC RESULT}

The proof of Theorem (1.2) from Theorem (1.1) closely follows the proof of the special case given in $[\mathrm{KM}]$, where the range shift of finite type $\Sigma_{B}$ is the full $n$-shift.

We first give the part of the argument borrowed directly from [KM]. Lemmas (4.1) and (4.2) are a new complication.

Let $m$ be a resolving block for the canonical right cover $\pi_{R}: \Sigma_{R} \rightarrow S$ (all paths $w$ in $\mathscr{G}_{R}$ with $\pi_{R}(w)=m$ end in the same state). Since $\pi_{R}$ is right resolving and left closing, any point in $S$ that contains an occurrence of $m$ has a single preimage under $\pi_{R}$. If we define the sofic system $T \subseteq S$ by

$$
T=\{y \in S: \text { the word } m \text { does not occur in } y\},
$$

then the points of $S$ with more than one preimage under $\pi_{R}$ are all in $T$. 
Now the preimage of $T$ under $\pi_{R}$, call it $\Sigma=\pi_{R}^{-1}(T) \subseteq \Sigma_{A}$ is a shift of finite type because it is obtained from $\Sigma_{A}$ by deleting any point of $\Sigma_{A}$ containing a word with $\pi_{R}$ image $m$. The shift of finite type $\Sigma$ contains all points $x$ in $\Sigma_{A}$ with at least two points in $\pi_{R}^{-1} \pi_{R}(x)$.

Using Lemmas (4.1) and (4.2) below, we construct a right-closing map $\psi: T$ $\rightarrow \Sigma_{B}$ and then define $\tilde{\varphi}: \Sigma \rightarrow \Sigma_{B}$ by

$$
\widetilde{\varphi}=\psi \circ\left(\left.\pi_{R}\right|_{\Sigma}\right) .
$$

We then use the extension Theorem (1.1) to extend $\tilde{\varphi}$ to a right-closing map $\varphi: \Sigma_{R} \rightarrow \Sigma_{B}$. Finally we define $\pi: S \rightarrow \Sigma_{B}$ by $\pi=\varphi \circ \pi_{R}^{-1}$. The map $\pi$ is well defined, for if $x, x^{\prime} \in \Sigma_{R}$ are distinct points with $\pi_{R}(x)=\pi_{R}\left(x^{\prime}\right)$, then $x, x^{\prime} \in \Sigma$ and

$$
\varphi(x)=\widetilde{\varphi}(x)=\psi\left(\pi_{R}(x)\right)=\psi\left(\pi_{R}\left(x^{\prime}\right)\right)=\widetilde{\varphi}\left(x^{\prime}\right)=\varphi\left(x^{\prime}\right) .
$$

By a compactness argument, $\pi$ is a block map. Also, $\pi$ never identifies two left asymptotic points in $S$ whose common left tail contains an occurrence of the resolving block $m$ because their (unique) preimages under $\pi_{R}$ are themselves left asymptotic in $\Sigma_{R}$, and $\varphi: \Sigma_{R} \rightarrow \Sigma_{B}$ is right closing. Thus $\pi$ is right closing almost everywhere.

The only remaining job is to construct a right-closing map $\psi: T \rightarrow \Sigma_{B}$. To do this we need two technical lemmas.

(4.1) Lemma. Let $T$ be a sofic system and let $\Gamma$ be an aperiodic shift of finite type with $h(T)<h(\Gamma)$ and $T \stackrel{\text { per }}{\rightarrow} \Gamma$. Then there is a shift of finite type $\Lambda$ with $T \subseteq \Lambda, h(\Lambda)<h(\Gamma)$, and $\Lambda \stackrel{\text { per }}{\rightarrow} \Gamma$.

Proof. Define $\Lambda_{n}$ to be the shift of finite type whose allowed $n$ blocks are all the $n$ blocks occurring in points of $T$. Note that $T \subseteq \Lambda_{n}$.

At this point we prove a claim to be used only in this proof.

(4.2) Claim. For all $L>0$, there exists $N>0$ such that for all $n \geq N$ and for all $l \leq L, \Lambda_{n}$ and $T$ have the same points of period $l$.

Proof of Claim. Let $\pi_{R}: \Sigma_{R} \rightarrow T$ be the canonical right cover of $T$ and let $d$ be the number of states of $\mathscr{G}_{R}$. We show that $\Lambda_{(d+1) L}$ and $T$ have the same points of period at most $L$ as follows. Let $y \in \Lambda_{(d+1) L}$ be a point of period $l \leq L$. Denote

$$
{ }_{0}(y)_{l-1}=y_{0} y_{1} \cdots y_{l-1}=c .
$$

There is a $(d+1) L$ path $w=w_{0} w_{1} \cdots w_{(d+1) L-1}$ in $\mathscr{G}_{R}$ that is $\pi_{R}$-labelled by $0(y)_{(d+1) L-1}=c^{m} r$, where $m=\lfloor(d+1) L / l\rfloor \geq d+1$ and $|r|<l$. Now the states $w_{0}, w_{l}, \ldots, w_{(m-1) l}$ in $\mathscr{G}_{R}$ cannot all be distinct since $m>d$, so $w_{i l}=w_{j l}$ for some $i, j$ with $0 \leq i<j \leq m-1$, and there is a cycle through the state $w_{i l}$ of $\mathscr{G}_{R}$ that is $\pi_{R}$-labelled by $c^{j-i}$. Thus the periodic point $y$ is an element of $\pi_{R}\left(\Sigma_{R}\right)=T$. This establishes the claim.

We finish the proof of Lemma (4.1). Because the shift of finite type $\Gamma$ is aperiodic, there is $L>0$ such that for all $l \geq L, \Gamma$ has a point of period $l$. For this $L$, choose $N$ as in the above claim, and fix $n \geq N$; the periodic points of period at most $L$ in $\Lambda_{n}$ and $T$ coincide. Now $T \stackrel{\text { per }}{\rightarrow} \Gamma$, so for each point $y$ of $\Lambda_{n}$ of period $p \leq L$, there is a point in $\Gamma$ whose period divides $p$. Also, by the choice of $L$, for each periodic point in $\Lambda_{n}$ of period exceeding 
$L$, there is a point in $\Gamma$ of equal period. Thus $\Lambda_{n} \stackrel{\text { per }}{\rightarrow} \Gamma$ for all $n \geq N$. Since $h(T)<h(\Gamma)$ and $h\left(\Lambda_{n}\right) \rightarrow h(T)$ as $n \rightarrow \infty$, we can choose $n \geq N$ such that $h\left(\Lambda_{n}\right)<h(\Gamma)$ and set $\Lambda=\Lambda_{n}$.

(4.3) Lemma. Let $\Lambda$ and $\Gamma$ be shifts of finite type with $\Gamma$ aperiodic, $h(\Lambda)<$ $h(\Gamma)$, and $\Lambda \stackrel{\text { per }}{\rightarrow} \Gamma$. Then there is a right-closing map $\widetilde{\psi}: \Lambda \rightarrow \Gamma$.

Proof. Following [B], denote by $\pi_{j}(\Sigma)$ the number of periodic points having least period $j$ in a shift of finite type $\Sigma$. Since $h(\Lambda)<h(\Gamma)$ there is some $L>0$ such that $\pi_{l}(\Lambda) \leq \pi_{l}(\Gamma), l \geq L$. Because $\Lambda \stackrel{\text { per }}{\rightarrow} \Gamma$, for each $l>0$, if $\pi_{l}(\Lambda)>0$, then $\pi_{q(l)}(\Gamma)>0$ for some $q(l)$ with $q(l) \mid l$.

At this point, we use [B, Lemma (2.1)]. We state the lemma here.

(4.4) Lemma [B, Lemma (2.1)]. Suppose $\Gamma$ is an irreducible shift of finite type of positive entropy and $\pi_{q}(\Gamma)>0$, and $M_{0}, M_{1}, \ldots, M_{k}$ are positive integers. Then there is an irreducible shift of finite type $\widehat{\Gamma}$ such that

(i) there is a right-closing factor map $\widehat{\varphi}: \widehat{\Gamma} \rightarrow \Gamma$,

(ii) if $\Gamma$ is aperiodic, then $\widehat{\Gamma}$ is aperiodic,

(iii) $\pi_{j}(\widehat{\Gamma})=\pi_{j}(\Gamma)$ if $j$ is not among $q, q M_{0}, \ldots, q M_{k}$,

(iv) $\pi_{q}(\widehat{\Gamma})=\pi_{q}(\Gamma)-q+q\left|\left\{i: M_{i}=1\right\}\right|$,

(v) $\pi_{q M_{i}}(\widehat{\Gamma})=\pi_{q M_{i}}(\Gamma)+q M_{i}\left|\left\{j: M_{j}=M_{i}\right\}\right|$, for those $i$ with $M_{i}>1$.

As remarked in $[\mathrm{B}], \widehat{\varphi}: \widehat{\Gamma} \rightarrow \Gamma$ as constructed there is right closing.

For each $1 \leq l<L$, apply the lemma iteratively, at the $l$ th stage 'blowing up' a periodic orbit of least period $q(l)$ into $\pi_{l}(\Lambda)$ periodic points of least period $l$. Specifically, set $M_{0}=1, M_{1}=M_{2}=\cdots=M_{\pi_{l}(\Lambda) / l}=l / q(l)$. The net effect of this is to construct a shift of finite type $\widehat{\Gamma}$ satisfying

(i) there is a right-closing factor map $\widehat{\varphi}: \widehat{\Gamma} \rightarrow \Gamma$,

(ii) $\hat{\Gamma}$ is aperiodic,

(iii) $\pi_{l}(\widehat{\Gamma})=\pi_{l}(\Gamma) \geq \pi_{l}(\Lambda), l \geq L$,

(iv) $\pi_{l}(\widehat{\Gamma})=\pi_{l}(\Gamma)+\pi_{l}(\Lambda), 1 \leq l<L$.

In particular, $\pi_{l}(\widehat{\Gamma}) \geq \pi_{l}(\Lambda)$ for $l \geq 1$. This, together with $h(\widehat{\Gamma})=h(\Gamma)>$ $h(\Lambda)$, allows us to use the Embedding Theorem [Kr2, Theorem 3] to conclude that there is an embedding $\theta: \Lambda \rightarrow \widehat{\Gamma}$. We now define $\tilde{\psi}: \Lambda \rightarrow \Gamma$ by $\widetilde{\psi}=\widehat{\varphi} \circ \theta$. Since $\theta$ is 1 -to- 1 and $\widehat{\varphi}$ is right closing, $\widetilde{\psi}$ is right closing also.

Recall that to prove Theorem (1.2), it remains to construct a right-closing map $\psi: T \rightarrow \Sigma_{B}$ using Lemmas (4.1) and (4.3).

Now

$$
h(T)=h(\Sigma)<h\left(\Sigma_{R}\right)=h(S)=h\left(\Sigma_{B}\right) .
$$

The inequality holds because the shift of finite type $\Sigma \subseteq \Sigma_{R}$ is obtained from the irreducible shift of finite type $\Sigma_{R}$ by disallowing the blocks whose $\pi_{R}$ image is the resolving block $m$. Also $T \stackrel{\text { per }}{\rightarrow} \Sigma_{B}$ because $T \subseteq S$ and $S \stackrel{\text { per }}{\rightarrow} \Sigma_{B}$. Thus Lemma (4.1) gives a shift of finite type $\Lambda$ with $T \subseteq \Lambda, h(\Lambda)<h\left(\Sigma_{B}\right)$, and $\Lambda \stackrel{\text { per }}{\rightarrow} \Sigma_{B}$. Now Lemma (4.3) gives a right closing map $\widetilde{\psi}: \Lambda \rightarrow \Sigma_{B}$. Finally, we define $\psi: T \rightarrow \Sigma_{B}$ as $\psi=\left.\widetilde{\psi}\right|_{T}$. This completes the proof of Theorem (1.2). 


\section{AN EXAMPLE}

Let $S \subseteq\{0,1\}^{\mathbb{Z}}$ be sofic system defined as follows. Between any two 1's in a point of $S$, there occurs an even number of 0 's. The sofic system $S$ is known as the even system.

The canonical right cover of $S$ is the golden mean system $\Sigma_{A}$ defined by the graph $\mathscr{G}_{A}$ depicted in Figure 1.

The 1-block canonical covering map $\pi_{R}: \Sigma_{A} \rightarrow S$ is given by $\pi_{R}(a)=1$, $\pi_{R}(b)=\pi_{R}(c)=0$. Since $\pi_{R}$ is left resolving, $S$ is almost finite type. Since $\Sigma_{A}$ has periodic points of all orders, we have $S \stackrel{\text { per }}{\rightarrow} \Sigma_{A}$. By Theorem (1.2) there is a 1-to-1 a.e. right-closing a.e. factor map $\pi: S \rightarrow \Sigma_{A}$. We will construct a specific $\pi$ following the construction outlined in the proofs of Theorems (1.1) and (1.2).

The only points $x$ in $\Sigma_{A}$ with $\left|\pi_{R}^{-1} \pi_{R}(x)\right|>1$ are the two points in the orbit of $(b c)^{\infty} ; \pi_{R}$ maps both of these points to $0^{\infty} \in S$. This is because the symbol 1 is a resolving block for $\pi_{R}$. Define $T \subseteq S$ as $T=\left\{0^{\infty}\right\}$ and $\Sigma \subseteq \Sigma_{A}$ as $\Sigma=\pi_{R}^{-1}(T)=\left\{(b c)^{\infty},(c b)^{\infty}\right\}$. In this example, the construction of the right-closing map $\psi: T \rightarrow \Sigma_{A}$ is easy (in fact there is only one such map). We define $\psi\left(0^{\infty}\right)=a^{\infty}$. We define $\tilde{\varphi}: \Sigma \rightarrow \Sigma_{A}$ by $\tilde{\varphi}=\psi \circ\left(\left.\pi_{R}\right|_{\Sigma}\right)$, so $\widetilde{\varphi}\left((b c)^{\infty}\right)=\widetilde{\varphi}\left((c b)^{\infty}\right)=a^{\infty}$.

We follow the construction of Theorem (1.1) in extending $\widetilde{\varphi}: \Sigma \rightarrow \Sigma_{A}$ to $\varphi: \Sigma_{A} \rightarrow \Sigma_{A}$. Now $A I=I A$ and $A I=A$, so we can take $S=I$ and $R=A$. Since $S=I$, we have $w(b)=(0,1,0)$ and $w(c)=(0,0,1)$.

Now $w(b)$ does not dominate $e_{\widetilde{\varphi}(b)}=e_{\widetilde{\varphi}(c)}=(1,0,0)$ (neither does $w(c)$ ), so it is impossible to split $\mathscr{G}_{A}$ with masses given by $I$ by $\widetilde{\varphi}$-respecting splittings into lean states. Notice $A^{2}$ is the lowest possible power of $A$ giving each state along the 2 cycle in $\mathscr{G}_{A}$ enough mass to split them both into lean states by rounds of $\widetilde{\varphi}$-respecting splittings. We have

$$
w(b)=(1,1,0) \geq(1,0,0), \quad w(c)=(1,1,1) \geq(1,0,0)
$$

Figure 2 illustrates 3 rounds of $\widetilde{\varphi}$-respecting splittings leading from $\mathscr{G}_{A}$ (with masses given by $A^{2}$ ) to a graph $\mathscr{G}_{A^{\prime}}$ with all lean states. States of $\mathscr{G}_{A^{\prime}}$ with a common ancestor in $\mathscr{G}_{A}$ are in the same column; states of $\mathscr{G}_{A^{\prime}}$ with a common elementary mass are in the same row.

The map $\varphi: \Sigma_{A} \rightarrow \Sigma_{A}$ is defined by $\mu \circ \alpha^{-1}$, where $\alpha: \Sigma_{A^{\prime}} \rightarrow \Sigma_{A}$ is the ancestor map (vertical projection in Figure 2) and $\mu: \Sigma_{A^{\prime}} \rightarrow \Sigma_{A}$ is the mass map (horizontal projection in Figure 2). Notice that the 2-cycle of $\Sigma_{A}$ maps by $\varphi$ onto the 1-cycle of $\Sigma_{A}$.

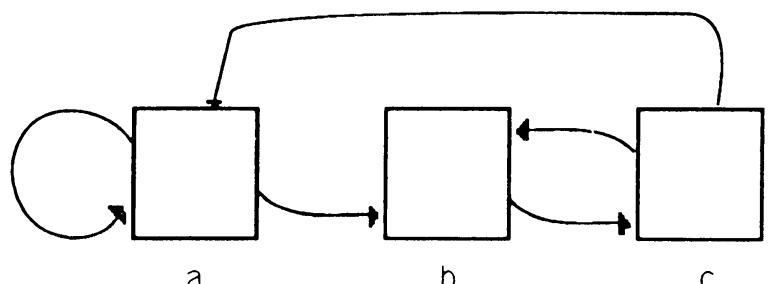

FIGURE 1 

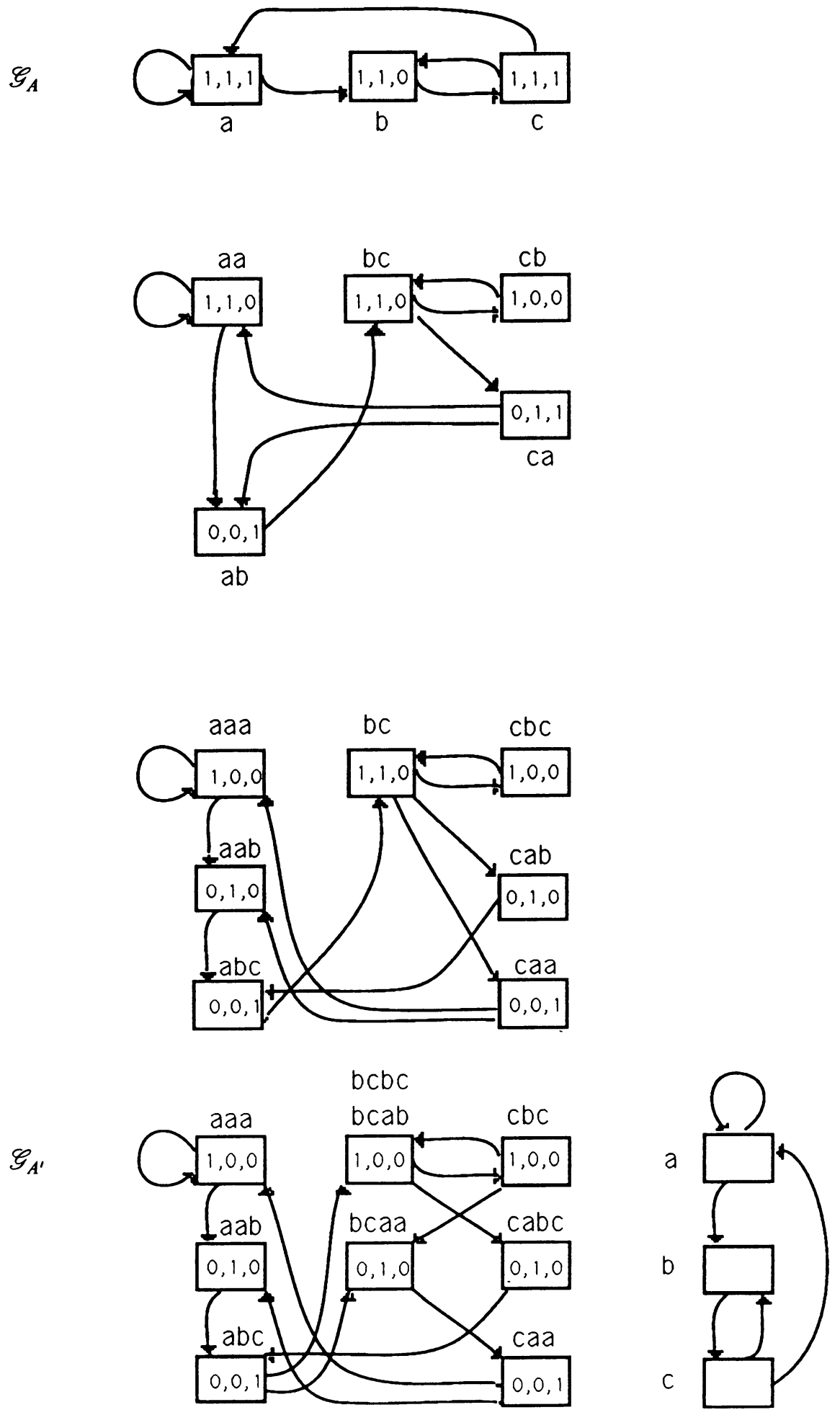

FIGURE 2 
TABLE 1

\begin{tabular}{|c|c|c|c|}
\hline & $\pi_{R}$ & & $\varphi$ \\
\hline 111 & $\longleftrightarrow$ & $a a a$ & $\longmapsto$ \\
\hline 110 & $\longleftrightarrow$ & $a a b$ & $\longmapsto$ \\
\hline 100 & $\longleftrightarrow$ & $a b c$ & $\longmapsto$ \\
\hline 0011 & $\longleftrightarrow$ & bcaa & $\longmapsto$ \\
\hline 0010 & $\longleftrightarrow$ & $b c a b$ & $\longmapsto$ \\
\hline 0000 & $\longleftrightarrow$ & $b c b c$ & $\longmapsto$ \\
\hline 011 & $\longleftrightarrow$ & $c a a$ & $\longmapsto$ \\
\hline 0100 & $\longleftrightarrow$ & $c a b c$ & $\longmapsto$ \\
\hline & & & $\longrightarrow$ \\
\hline
\end{tabular}

The map $\varphi: \Sigma_{A} \rightarrow \Sigma_{A}$ is the 4-block map defined by the last two columns of Table 1. Each block $w$ listed in the center column of Table 1 is contained in a state of $\mathscr{G}_{A^{\prime}}$.

The table should be interpreted as follows. If $x \in \Sigma_{A}$ has $0(x)_{|w|-1}=w$ for a word $w$ listed in the second column, then ${ }_{0}(\varphi(x))_{0}$ is the corresponding symbol listed in the last column.

The first column of Table 1 gives the $\pi_{R}$ images of the corresponding words in the second column. Each word in the first column except words 0000 and 000 has a unique $\pi_{R}$ preimage (moreover, it appears in the second column). If $x \in S$ has $0(x)_{2}=000$, then $0\left(\pi_{R}^{-1} x\right)_{2}$ is either $b c b$ or $c b c$; and if $y \in \Sigma_{A}$ has $0(y)_{2}=b c b$ or $c b c$, then $0(\varphi y)_{0}=a$. Hence, if $0(x)_{2}=000$, then ${ }_{0}(\pi x)_{0}=a$. Thus $\pi: S \rightarrow \Sigma_{A}$ is a 4-block map and can be computed by reading from the first column of Table 1 all the way over to the third column.

By [BMT, Proposition 4.12], if $\pi: S \rightarrow \Sigma$ is a right-closing factor map from a sofic system $S$ onto a shift of finite type $\Sigma$, then $S$ is actually a shift of finite type. Thus, the map $\pi: S \rightarrow \Sigma_{A}$ constructed above cannot be right closing everywhere. We can see this directly by observing that $\pi\left(0^{\infty} \underline{1} 0^{\infty}\right)=\pi\left(0^{\infty} \underline{0} 1^{\infty}\right)=$ $a^{\infty} b \underline{c} a^{\infty}$, where the underscored symbols occur at time 0 .

\section{REFERENCES}

[AM] R. Adler and B. Marcus, Topological entropy and equivalence of dynamical systems, Mem. Amer. Math. Soc., no. 219, 1979.

[A1] J. Ashley, Bounded-to-1 factors of an aperiodic shift of finite type are 1-to-1 almost everywhere factors also, Ergodic Theory Dynamical Systems 10 (1990), 615-625.

[A2] _ Resolving factor maps for shifts of finite type with equal entropy, Ergodic Theory and Dynamical Systems 11 (1991), 219-240.

[B] M. Boyle, Lower entropy factors of sofic systems, Ergodic Theory and Dynamical Systems 4 (1984), 541-557.

[BMT] M. Boyle, B. Marcus, and P. Trow, Resolving maps and the dimension group for shifts of finite type, Mem. Amer. Math. Soc., no. 377, 1987.

[KM] R. Karabed and B. Marcus, Sliding-block coding for input-restricted channels, IEEE Trans. Inform. Theory 34 (1988), 2-26.

[Kr1] W. Krieger, On dimension functions and topological Markov chains, Invent. Math. 56 (1980), 239-250.

[Kr2] _ On the subsystems of topological Markov chains, Ergodic Theory Dynamical Systems 2 (1982), 195-202. 
[M] B. Marcus, Factors and extensions of full shifts, Monatsh. Math. 88 (1979), 239-247.

[S] E. Seneta, Non-negative matrices and Markov chains, 2nd ed., Springer-Verlag, New York, 1981.

[We] B. Weiss, Subshifts of finite type and sofic systems, Monatsh. Math. 77 (1973), 462-474.

[Wi] R. Williams, Classification of shifts of finite type, Ann. of Math. 98 (1973), 120-153; Errata, Ann. of Math. 99 (1974), 380-381.

Department of Mathematics, University of Washington, Seattle, Washington 98195 Current address: IBM Research Division, Almaden Research Center, 650 Harry Road, San Jose, California 95120-6099

E-mail address: jashley@almaden.ibm.com 